\title{
Dance of the SNAREs: Assembly and Rearrangements Detected with FRET at Neuronal Synapses
}

\author{
Vadim Degtyar, Ismail M. Hafez, Christopher Bray, and Robert S. Zucker \\ Department of Molecular and Cell Biology, University of California, Berkeley, Berkeley, California 94720
}

Soluble $N$-ethylmaleimide-sensitive fusion protein attachment protein receptors (SNAREs) mediate vesicle fusion with the plasma membrane on activation by calcium binding to synaptotagmin. In the present study, we used fluorescence resonance energy transfer (FRET) and fluorescence lifetime imaging microscopy between fluorescently labeled SNARE proteins expressed in cultured rat hippocampal neurons to detect resting SNARE complexes, their conformational rearrangement on exocytosis, their disassembly before endocytosis of vesicular proteins, and SNARE assembly at newly docked vesicles. Assembled SNAREs are not only present in docked vesicles; unexpected residual "orphan SNARE complexes" also reside in para-active zone regions. Real-time changes in FRET between $\mathrm{N}$-terminally labeled SNAP-25 and VAMP reported a reorientation of the SNARE motif upon exocytosis, SNARE disassembly in the active zone periphery, and SNARE reassembly in newly docked vesicles. With VAMP labeled C-terminally, decreased fluorescence in C-terminally labeled syntaxin (extracellular) reported trans-cis-conformational changes in SNAREs on vesicle fusion. After fusion SNAP-25 and syntaxin disperse along with VAMP, as well as the FRET signal itself, indicating diffusion of intact SNAREs after vesicle fusion but before their peripheral disassembly. Our measurements of spatiotemporal dynamics of SNARE conformational changes and movements refine models of SNARE function. Technical advances required to detect tiny changes in fluorescence in small fractions of labeled proteins in presynaptic boutons on a time scale of seconds permit the detection of rapid intermolecular interactions between small proportions of protein partners in cellular subcompartments.

\section{Introduction}

The docking and fusion of vesicles with the plasma membrane requires the assembly of a vesicle protein [synaptobrevin or vesicle-associated membrane protein (VAMP)] and two plasma membrane proteins [syntaxin and synaptosomal associated protein of $25 \mathrm{kDa}$ (SNAP-25)] into a tight protein complex called the SNARE complex. SNARE is an acronym for SNAP receptor, where SNAP stands for soluble $N$-ethylmaleimide (NEM)sensitive fusion protein (NSF) attachment protein. The name SNARE recalls its disassembly before endocytosis by the action of $\alpha$-SNAP and NSF to allow the recovery of VAMP with vesicular membrane while leaving syntaxin and SNAP- 25 on the plasma

\footnotetext{
Received May 14, 2012; revised Jan. 22, 2013; accepted Jan. 25, 2013.

Author contributions:V.D., I.M.H., and R.S.Z. designed research, V.D. and I.M.H. performed research, V.D., I.M.H. and R.S.Z. analyzed data, and R.S.Z. wrote the paper.

This research was supported by the National Institutes of Health (Grants R21 NS048016 and R01 NS054760) and the National Science Foundation (Grant SGER 0332968). We thank Ed Levitan, Ed Chapman, Richard Kramer, Ehud Isacoff, Manfred Lindau, and Doris Fortin for discussion, Russell English for technical assistance, Christophe HansenEstruch for assistance with data analysis, Arunesh Saras and Sarit Ahituv for assistance with molecular biology and for advice, Ed Chapman for providing EYFP-SNAP-25B and ECFP-VAMP-2, Roger Tsien for the gift of citrine, David Piston for the gift of monomeric cerulean, and Mark Rizzo for the gifts of cerulean-3-VAMP-2 and agarose beads coated with cerulean-venus or cerulean-3-venus dimers, and Kevin Staras for help in developing the initial protocols and performing the preliminary experiments.

The authors declare no competing financial interests.

Correspondence should be addressed to Robert S. Zucker, Department of Molecular and Cell Biology, 111 Life Sciences Addition, University of California, Berkeley, Berkeley, CA 94720. E-mail: zucker@berkeley.edu.

I. M. Hafez's present address: Brain Research Centre, University of British Columbia, Vancouver, BC V6T 2B5, Canada.

C. Bray's present address: Accuray, Sunnyvale, CA 94089.

DOI:10.1523/JNEUROSCI.2337-12.2013

Copyright $\odot 2013$ the authors $\quad 0270-6474 / 13 / 335507-17 \$ 15.00 / 0$
}

membrane to be reused in the fusion of newly docked vesicles (Chen and Scheller, 2001). SNAREs themselves are competent to catalyze the fusion of vesicle and plasma membranes (Weber et al., 1998; Domanska et al., 2009), but the sudden fusion of vesicles after action potentials requires the binding of calcium ions to $\mathrm{C} 2$ domains of synaptotagmin (Tucker et al., 2004), which interact in turn with SNARE proteins and plasma membrane phospholipids to greatly accelerate the fusion process.

The exact sequence of molecular events underlying membrane fusion remains unknown. Extensive biochemical evidence suggests that the priming of vesicles for fusion involves the final assembly of SNAREs into a tight coiled-coil complex of the N termini of VAMP, syntaxin, and SNAP-25, whereas the $\mathrm{C}$ termini of VAMP and syntaxin and the intercoiled region of SNAP-25 are embedded in their respective membranes. Therefore, the primed SNARE complex is believed to be a trans-membrane complex that provides some of the tension needed for membrane fusion to occur. A variety of other cytoplasmic and vesicle proteins bind to SNAREs and regulate their function (Brunger, 2006); the SNAREs alone are sufficient to form a fusion pore between adjacent membranes (Han et al., 2004), whereas synaptotagmin is needed to endow calcium sensitivity and rapid kinetics to the process (Tucker et al., 2004).

Current models assume rearrangements in the SNARE partners as fusion pores form and vesicle and plasma membranes fuse. After fusion, the $\mathrm{N}$ termini of all SNARE proteins remain together, whereas the previously intravesicular $\mathrm{C}$ terminus of VAMP joins that of syntaxin on the extracellular surface in a new cis-configuration. In this study, we used two labeling schemes to 
reveal these molecular rearrangements: In the first, we attached the green fluorescent protein (GFP)-like fluorophores cerulean (or cerulean-3) and citrine to the N termini of VAMP and SNAP25 , respectively (Fig. 1a), in rat cultured hippocampal neurons and used fluorescence resonance energy transfer (FRET) to detect changes in their separation and orientation occurring after stimulated secretion. Previous studies have shown that their $\mathrm{N}$ termini are closely apposed in the SNARE complex, and fluorophores attached there are capable of displaying FRET (Lin and Scheller, 1997; Sutton et al., 1998; Xia et al., 2001). In the second scheme, we attached citrine and cerulean to the $\mathrm{C}$ termini of VAMP and syntaxin, respectively (see Fig. 10a), and used FRET to report a trans-cis-conformational change in SNAREs on vesicle fusion.

\section{Materials and Methods}

Molecular biology. Cerulean or its photostable variant cerulean-3 and citrine were selected as fluorophores that are brighter, are less subject to photobleaching, and FRET more efficiently than enhanced cyan and yellow fluorescent proteins (ECFP and EYFP) (Griesbeck et al., 2001; Rizzo et al., 2004; Markwardt et al., 2011). GFPs attached to proteins can dimerize to produce an artifactual FRET even when the protein partners do not interact, so this was prevented by using monomeric citrine and cerulean (Zacharias et al., 2002).

Rat SNAP-25B labeling started with EYFP-SNAP-25B in the pEYFP-C1 (Clontech) vector. Citrine was made monomeric by introducing the A206K mutation using the Stratagene QuikChange SiteDirected Mutagenesis Kit, and exchanged with EYFP by digestion with restriction endonucleases after ligation. For rat VAMP-2 N-terminal labeling, the ECFP of ECFP-VAMP-2 in pECFP-C1 was exchanged for monomeric cerulean. VAMP-2 was C-terminally labeled by replacing EYFP on VAMP-2-EYFP in pEYFP-N1 with monomeric citrine. Finally, rat syntaxin-1A was C-terminally labeled by replacing ECFP on syntaxin-1A-ECFP in pECFP-N1 with monomeric cerulean. FRET of $\mathrm{N}$-terminally labeled constructs was further optimized by cutting out the 12-aa linker regions with BglII and EcoR I endonucleases, shortening the 5 ' overhangs with mung bean nuclease, followed by blunt-end religation to produce the in-frame short but rotationally flexible SGLT linker directly between the $\mathrm{C}$-terminal $\mathrm{K}$ of citrine and the $\mathrm{N}$-terminal $\mathrm{M}$ of SNAP-25B. VAMP-2 N-terminally linked in the same fashion with the same four amino acids to cerulean-3 was kindly provided by Mark Rizzo (University of Maryland, College Park, MD). Botulinum E toxin resistance was conferred on mCit-4aa-SNAP-25B by introducing a D179K mutation into SNAP-25B (Finley et al., 2003), whereas botulinum C1 toxin resistance was conferred by an A199R mutation and tetanus toxin resistance was conferred on mCer-4aa-VAMP-2 by introducing Q76V and F77W mutations into VAMP-2 (Regazzi et al., 1996).

Cell culture, transfection, and intoxication. Hippocampi were removed from embryonic day 18-21 rat fetuses of either sex into HBSS without calcium or magnesium plus $20 \mathrm{~mm}$ glucose and $10 \mathrm{~mm}$ HEPES, digested for $20 \mathrm{~min}$ at $37^{\circ} \mathrm{C}$ in $0.25 \%$ trypsin, and then rinsed in HBSS. Cells were dissociated by triturating four times in culture medium through a set of fire-polished Pasteur pipettes and strained through a $45 \mu \mathrm{m}$ mesh into 32 $\mathrm{ml}$ of medium. Medium contained $190 \mathrm{ml}$ of Earle's MEM without phenol red or glutamine, $0.75 \mathrm{~g}$ of glucose, $10 \mathrm{ml}$ of fetal bovine serum (FBS), and $200 \mu \mathrm{l}$ of MITO + serum extender (BD Biosciences) and was filtered and stored at $4^{\circ} \mathrm{C}$. To this medium, $0.5 \mathrm{ml}$ of $200 \mathrm{~mm}$ L-glutamine and 1 $\mathrm{ml}$ of B27 supplement (50×; Invitrogen) were added before use. Cells were plated at $1.5 \mathrm{ml}$ per $35 \mathrm{~mm}$ culture dish on a $25 \mathrm{~mm}$ Bellco coverslip, precleaned by sonicating $1 \mathrm{~h}$ each in concentrated $\mathrm{HNO}_{3}$ and $\mathrm{HCl}$, washed, racked, and coated for several hours with poly-L-lysine $(0.5$ $\mathrm{mg} / \mathrm{ml}$ in $50 \mathrm{~mm}$ boric acid and $12.5 \mathrm{~mm} \mathrm{Na}$-tetraborate), then rinsed and dried. Cell density was $\sim 250 / \mathrm{mm}^{2}$.

After growing for $7 \mathrm{~d}$ at $37^{\circ} \mathrm{C}$ in $7 \% \mathrm{CO}_{2}$, cells were transfected by an efficient $\mathrm{Ca}_{3}\left(\mathrm{PO}_{4}\right)_{2}$ precipitation procedure (Jiang et al., 2004) in medium lacking FBS. For transfection, 2-4 $\mu \mathrm{g}$ of DNA in $33 \mu \mathrm{l}$ of $250 \mathrm{~mm}$ $\mathrm{CaCl}_{2}$ was added drop-wise in a clear polystyrene tube to $33 \mu \mathrm{l}$ of pre- warmed $2 \times$ HEPES-buffered salt solution composed of (in mM): 174 $\mathrm{NaCl}, 10 \mathrm{KCl}, 1.4 \mathrm{Na}_{2} \mathrm{HPO}_{4}, 15$ glucose, and 42 HEPES, pH 7.09, and mixed lightly. Approximately $66 \mu \mathrm{l}$ of labeled VAMP-2, SNAP-25B, or syntaxin-1A DNA, or a mixture of two constructs, was deposited gently under the transfection medium onto cells. After incubation at $37^{\circ} \mathrm{C} / 7 \%$ $\mathrm{CO}_{2}$ for $1 \mathrm{~h}$, transfection medium was replaced twice with culture medium and cells incubated for $1-5 \mathrm{~d}$ before use. SNAP-25B expression reaches a peak within $1-2 \mathrm{~d}$ and syntaxin-1A within $2-3 \mathrm{~d}$, whereas VAMP-2 expression is more vigorous and increases gradually for 1 week (Sankaranarayanan and Ryan, 2000; Li and Murthy, 2001). Efficient FRET requires similar expression levels and fluorescence intensities (Berney and Danuser, 2003), and this requirement plus minimizing mistargeting of VAMP-2 to the plasma membrane were met by cotransfecting cells with a [DNA] ratio of $6: 1$ for SNAP-25B or syntaxin-1A to VAMP-2. With N-terminal labeling, the brighter citrine was placed on the more weakly expressing SNAP-25B and conducted experiments 2-4 $\mathrm{d}$ after transfection. For C-terminal labeling, experiments were conducted $2-5 \mathrm{~d}$ after transfection. To minimize overexpression of SNAP$25 \mathrm{~B}$ or syntaxin- $1 \mathrm{~A}$ in vesicles, lighter transfections were used in singlelabel dispersion experiments, resulting in dimmer and noisier fluorescence records.

Bacterial toxins were loaded into cells by endocytic uptake (Matteoli et al., 1996; Keller et al., 2004). The culture medium was changed to a depolarizing solution of HEPES buffered salt solution containing $55 \mathrm{~mm}$ $\mathrm{KCl}$ with $\mathrm{NaCl}$ reduced to $37 \mathrm{~mm}$, plus either or both of tetanus toxin (10 nM; List Biological Laboratories) or activated nicked botulinum E toxin (25 nм; Wako Chemicals). Some cells were exposed to a similar depolarizing solution containing $25 \mathrm{~nm}$ botulinum $\mathrm{C} 1$ toxin (Metabiologics). After $4 \mathrm{~min}$, cells were incubated for $2 \mathrm{~h}$ in culture medium at $37^{\circ} \mathrm{C} / 7 \% \mathrm{CO}_{2}$.

Stimulation and FM4-64 staining. A coverslip was mounted in a field stimulation chamber (RC21-BRFS; Warner Instruments) clamped to a custom fixed platform, and viewed with an Olympus IX7 inverted microscope on a movable platform through a $60 \times 1.25$ numerical aperture (NA) or $40 \times 1.35 \mathrm{NA}$ oil objective. Cells were superfused at $0.3 \mathrm{ml} / \mathrm{min}$ at $25-28^{\circ} \mathrm{C}$ with external medium containing the following (in $\mathrm{mM}$ ): 119 $\mathrm{NaCl}, 2.5 \mathrm{KCl}, 2.5 \mathrm{MgCl}_{2}, 3 \mathrm{CaCl}_{2}, 25 \mathrm{HEPES}, \mathrm{pH} 7.4$, and 30 glucose; in some experiments, $10 \mu \mathrm{M}$ 6,7-dinitroquinoxaline-2,3-dione (A.G. Scientific) and $50 \mu \mathrm{M}$ DL-2-amino-5-phosphovaleric acid (Sigma) were added to block recurrent activity. Solutions were degassed under vacuum to minimize photobleaching of fluorescent dyes. Stimulation consisted of $8-9 \mathrm{~V} / \mathrm{mm}, 40-50 \mathrm{~mA}, 1 \mathrm{~ms}$ bipolar pulses to Pt wires, which was $50 \%$ above threshold as assessed by uniform FM4-64 staining of processes both parallel and perpendicular to the field lines. To stain endocytosed vesicles, $15 \mu \mathrm{M}$ FM4-64 (Invitrogen) was added at $3 \mathrm{ml} / \mathrm{min}$, and then cells were stimulated at $10 \mathrm{~Hz}$ for $60 \mathrm{~s}$ and rested for 2 min to allow endocytosis to complete (Ryan and Smith, 1995), followed by a $20-40$ min dye-free wash. Healthy transmission was confirmed by rapid destaining of well stained boutons within $10-12 \mathrm{~s}$ as measured during 30 $\mathrm{Hz}$ stimulation.

Fluorescence microscopy and image analysis. Fluorescence excitation was provided by a TILL Photonics Polychrome IV monochrometer. For examining cells and locating transmitting boutons of transfected neurons, fluorescence was excited at $473 \pm 5 \mathrm{~nm}$ with the Polychrome's white light pedestal removed using a $480 \pm 20 \mathrm{~nm}$ filter and a $505 \mathrm{~nm}$ dichroic mirror; emission was collected through a $515 \mathrm{~nm}$ long-pass filter. Optical components were from Chroma Technology. This arrangement excites cerulean, citrine, and FM4-64 fluorescence, with GFPs appearing green and FM4-64 fluorescence appearing red. In some experiments, the emission beam was split with an Optical Insights Dual View Micro-Imager using a $585 \mathrm{~nm}$ dichroic and $535 \pm 15$ and $660 \pm 20$ $\mathrm{nm}$ barrier filters, providing concurrent images of GFP and FM4-64 fluorescence to a Retiga EXi 1394 (QImaging) $1397 \times 1040$ pixel cooled CCD monochrome camera. Time-lapse dual-frame backgroundsubtracted images were collected at $0.2 \mathrm{~s}^{-1}$ and analyzed using Compix Simple PCI software, usually with $4 \times 4$ binning producing binned pixels of $430 \times 430 \mathrm{~nm}$.

For FRET measurements, the microscope filter cube was replaced with one containing a dual bandpass filter $(432 \pm 12$ and $502 \pm 12 \mathrm{~nm})$ to 
a

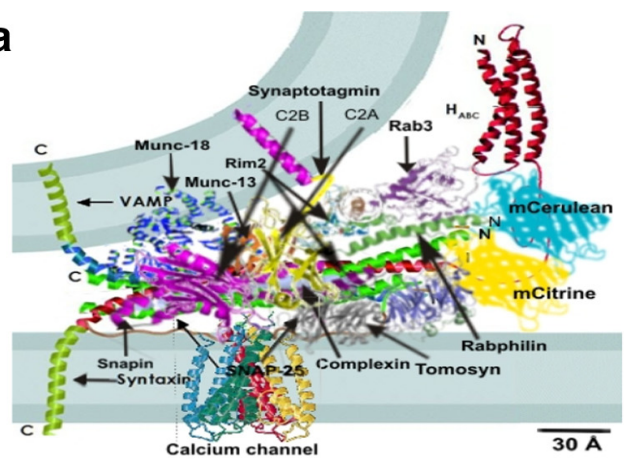

b

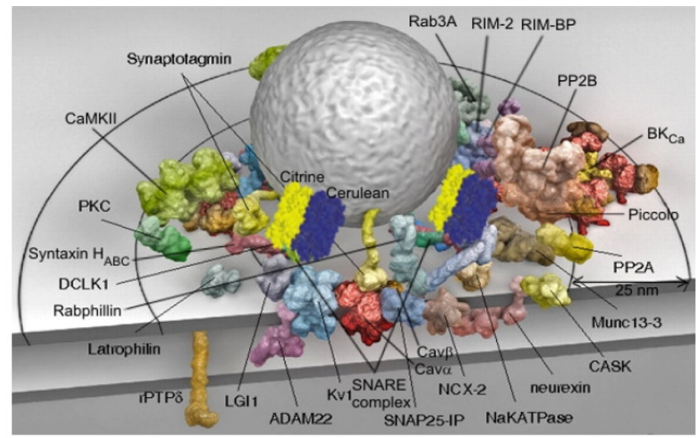

c

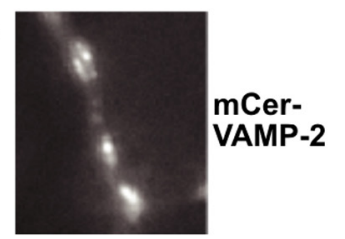

d

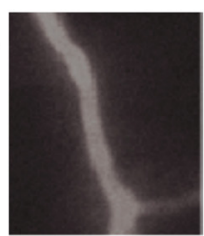

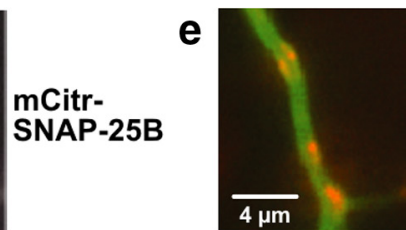

Red: $\mathrm{mCer}-$

VAMP-2

Green: mCitSNAP-25B

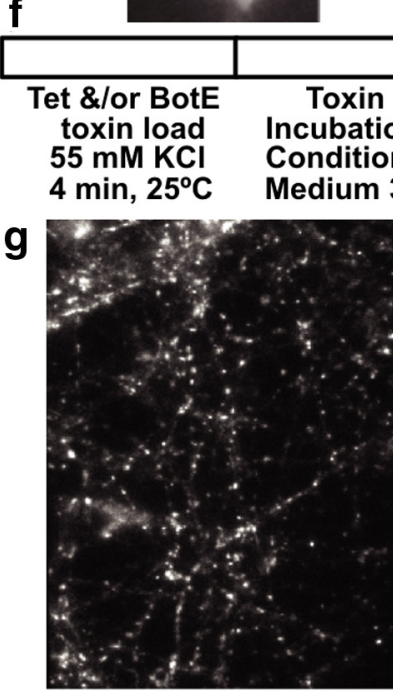

Normal FM4-64 staining on stimulation in controls

\begin{tabular}{cccc} 
& & & \\
\hline FM4-64 load & FM load & Wash & Destain \\
$10 \mathrm{~Hz}, 2 \mathrm{~min}$ & $2 \mathrm{~min}$ rest & $15 \mathrm{~min}$ & $10 \mathrm{~Hz}, 2 \mathrm{~min}$ \\
External Sol. & External Sol. & External Sol. & External Sol. \\
+CNQX/AP-5 & +CNQX/AP-5 & +CNQX/AP-5 & +CNQX/AP-5
\end{tabular}
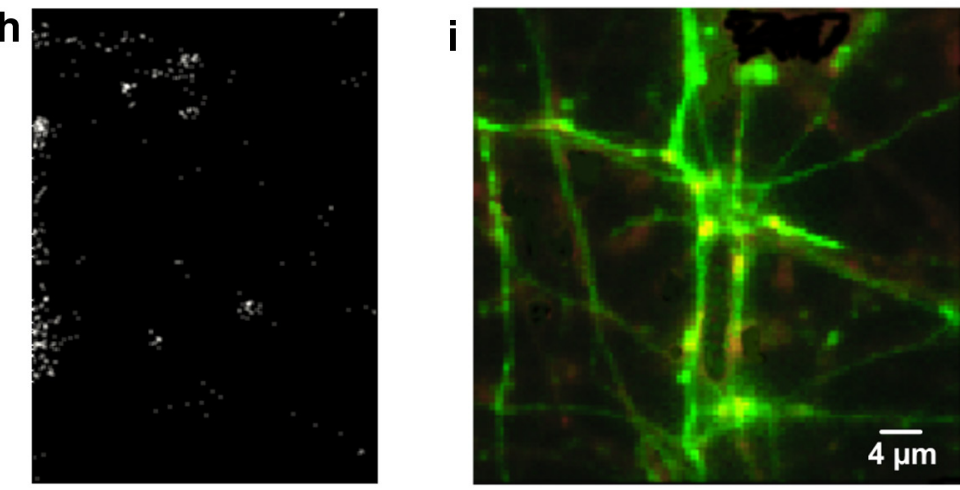

Lack of FM4-64 staining in FM4-64 staining (red) in toxin-resistant toxin-treated controls transfected cells (mCit-SNAP-25B, green)
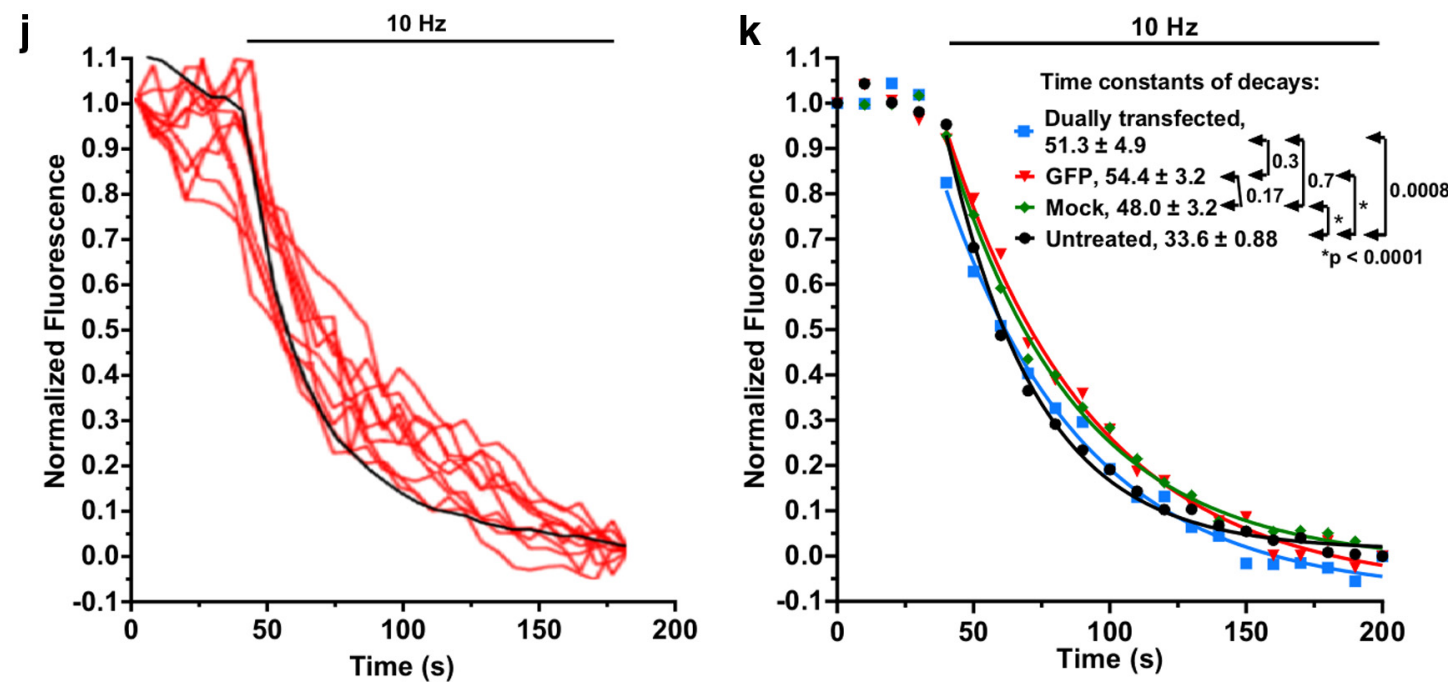

Figure 1. Functional expression of exogenous labeled SNARE proteins. $\boldsymbol{a}$, Schematic of assembled SNARE complex comprising VAMP labeled with cerulean, SNAP-25 labeled with citrine, and syntaxin with its $\mathrm{N}$-terminal $\mathrm{H}_{\mathrm{ABC}}$ moiety plus associated proteins involved in exocytosis. Ribbon diagrams are from published databases, drawn to scale, oriented with interacting surfaces touching. ( and N mark SNARE protein termini. $\boldsymbol{b}$, Protein-dense environment surrounding GFP-labeled SNAREs (adapted from Müller et al., 2010). $\boldsymbol{c}-\boldsymbol{e}$, Fluorescence images of a transfected neural axon showing correct localization of mCer-VAMP-2 (c), mCit-SNAP-25B (d), and pseudocolor overlay (e).f, Toxin treatment and FM staining/destaining protocol. $\boldsymbol{g}$, Synaptic vesicles load with FM4 - 64 during $10 \mathrm{~Hz}$ stimulation (bright puncta) in untreated cultures. $\boldsymbol{h}$, Toxin-treated cultures showing only diffuse background staining. $\boldsymbol{i}$, Pseudocolor overlay showing toxin-resistant $m$ Cer-VAMP-2 and mCit-SNAP-25B (green), with FM4 - 64 loading of vesicles on stimulation (red puncta, yellow when merged with green). Scale bar in $\boldsymbol{e}$ also applies to $\mathbf{c}$ and $\boldsymbol{d}$; scale bar in (Figure legend continues.) 
eliminate white light reflected by the monochrometer prism, a triple dichroic (reflecting 420-445, 500-520, and 610-640 nm and transmitting $460-490,530-600$, and $650-775 \mathrm{~nm}$ ) for cerulean and citrine plus FM4-64 excitation in the first two reflection bands, and separate cerulean, citrine, and FM4-64 emission in the three transmission bands. Emitted light was split by a Photometrics Quad View image splitter equipped with a $495 \mathrm{~nm}$ dichroic and a $470 \pm 10 \mathrm{~nm}$ barrier filter and a $560 \mathrm{~nm}$ dichroic with $532 \pm 12 \mathrm{~nm}$ barrier filter to obtain concurrent images of cerulean (donor) and citrine (acceptor) fluorescence, respectively. The third and fourth channels were separated by a $625 \mathrm{~nm}$ dichroic with a $595 \pm 10 \mathrm{~nm}$ barrier filter in channel 3 and a $>615 \mathrm{~nm}$ long-pass filter in channel 4, confining FM4-64 emission to the fourth channel. In some experiments, incident light filtered through a $580 \pm 10 \mathrm{~nm}$ barrier filter passed the microscope dichroic and was deflected into channel 3 to provide bright-field images. Excitation alternated between $435 \pm 5 \mathrm{~nm}$ and $505 \pm 5 \mathrm{~nm}$ from the Polychrome IV, with images generally acquired every 2 s using an Andor Technology Ixon DU-897 $512 \times$ 512-pixel, cooled electron multiplying CCD camera without binning with a pixel size of $267 \times 267 \mathrm{~nm}$. In this way, separate images of donor fluorescence $\left(I_{D}\right)$ and acceptor fluorescence on excitation of the donor $\left(I_{F}\right.$, an uncorrected FRET signal) in alternation with acceptor fluorescence $\left(I_{A}\right)$ simultaneously with FM-4-64 fluorescence and a bright-field image could be acquired. Image acquisition and Polychrome IV control relied upon Photometrics IQ software with postprocessing using NIH ImageJ. Measurements of SNAP-25B, syntaxin-1A, or VAMP-2 dispersion usually involved excitation of a single fluorophore (cerulean at $436 \mathrm{~nm}$ or citrine at $505 \mathrm{~nm}$ ), with imaging through an additional $1.5 \times$ magnifying lens rendering $178 \times 178 \mathrm{~nm}$ pixels. Likewise, only cerulean fluorescence was monitored for measuring resting FRET by donor dequenching after photobleaching citrine with $510 \mathrm{~nm}$ excitation for $\sim 10 \mathrm{~min}$ or until its fluorescence was reduced by $\sim 90 \%$.

FRET calculations. To correct sensitized FRET measurements for spurious acceptor excitation and donor emission spillover, some cells from each litter were transfected with just mCer-VAMP-2 and $\alpha$ was measured as $I_{F} / I_{A}$; and other cells were transfected with just mCit-SNAP-25B and $\beta$ was measured as $I_{F} / I_{D}$. The $\alpha$ value varied between 0.045 and 0.055 and $\beta$ between 0.25 and 0.30 . To discover the origins of this variability, preliminary experiments were performed on agarose beads coated with a cerulean-venus dimer separated by 8 aa. The fluorescence characteristics, and therefore $\alpha$ and $\beta$, were found to depend mildly on $\mathrm{pH}$ in the range of 6.9-7.4, ionic strength in the range of $125-175 \mathrm{~mm}$, refractive index of $1.38-1.43$ (30-70\% glycerol), extent of photobleaching, and relative fluorescence intensities of protein and bead material, the latter being analogous to cell autofluorescence. These properties are likely to vary within these ranges in cytoplasm among cells from different litters, and probably explain the need to measure $\alpha$ and $\beta$ in each set of cultures. These beads were also used to check the procedures for correcting FRET. They had a reported FRET efficiency of 0.47 , which was measured as 0.45 . The $\gamma$ $(0.081)$ and $\xi(0.64)$ were estimated from published absorbance, excitation, and emission spectra of citrine and cerulean (Griesbeck et al., 2001; Rizzo et al., 2004; Markwardt et al., 2011).

Fluorescence lifetime imaging microscopy. Fluorescence lifetime imaging microscopy (FLIM) measurements were made with a Zeiss 510 nonlinear optics Axiovert 200M laser scanning microscope in the Molecular Imaging Center at University of California, Berkeley. The microscope is equipped with a NeoFLUAR $40 \times 1.3 \mathrm{NA}$ oil objective and a Becker and Hickl SPC-730 Time-Correlated Single Photon Counting Module. Samples were excited by $436 \mathrm{~nm}$ laser light at $0.33-0.8 \mu \mathrm{W}$ generated by

(Figure legend continued.) $\quad \boldsymbol{i}$ also applies to $\boldsymbol{g}$ and $\boldsymbol{h} . \boldsymbol{j}$, FM4 -64 destained at nearly normal rates; red traces track single boutons of 10 dually toxin-resistant transfected cells treated with tetanus and botulinum $D$ toxins; black line is average of 10 untreated controls. $\boldsymbol{k}$, Neurons transfected with toxin-resistant SNAREs $(n=44)$ destain at the same rate as cells transfected with GFP $(n=48)$ or mock transfection controls $(n=48)$; untreated controls $(n=43)$ destain slightly faster, releasing a higher fraction of stained vesicles per action potential. Block shows time constants of exponential fits to fluorescence decays and probabilities of differences between groups (two-tailed $t$ tests). frequency-doubling the $872 \mathrm{~nm} 120-150$ fs pulses at $80 \mathrm{MHz}$ from a SpectraPhysics mode-locked Ti:Sapphire Tsunami laser using a SpectraPhysics 3980 Pulse Selector. Emitted light was filtered through a Chroma $480 \pm 10 \mathrm{~nm}$ bandpass filter and detected by a Becker and Hickl PMC100 photomultiplier tube coupled to the fiber-out port of the 510 LSM. Images of $128 \times 128$ or $256 \times 256$ pixels were acquired over $1-4 \mathrm{~min}$. For dequenching cerulean, citrine was photobleached with 320 scans of 514 $\mathrm{nm}$ excitation from an Ar laser at full power for $1.6 \mu$ s per pixel per scan. The apparent FRET ratio of the donor (product of the fraction of donor in the FRET-generating complex times the FRET efficiency of the complex), $F R_{D}$, was estimated from the difference in average donor lifetime change from its initial value $\left(\tau_{i}\right)$ on photobleaching the acceptor with and without acceptor present $(\Delta \tau)$ as $F R_{D}=\Delta \tau / \tau_{i}$.

Estimating fractions of proteins in SNAREs. FRET ratios of donor or acceptor report the fractions of donor or acceptor energy engaged as FRET and correspond to the product of the fraction of donor or acceptor molecules subjected to FRET times the FRET efficiency of the FRET pair. For closely apposed cerulean and citrine (Fig. 1 $a, b$ ), FRET efficiencies between 0.43 and 0.50 have been measured for cerulean/venus pairs separated by flexible 5 aa linkers (Griesbeck et al., 2001; Rizzo et al., 2004; Day et al., 2008). Citrine has a Förster distance slightly greater than that of Venus (Rizzo et al., 2006) and that of cerulean-3 is longer than for regular cerulean (Markwardt et al., 2011), allowing for an even greater FRET efficiency. Although the precise spatial relationship between the closely apposed $\mathrm{N}$ termini of SNAP-25B and VAMP-2 in a SNARE complex is uncertain, these considerations led us to assume a FRET efficiency of $\sim 0.5$ in the highly congested space surrounding assembled SNAREs, similar to that measured by Xia et al. (2001) between ECFP-VAMP-2 and EYFP-SNAP-25B in SNARE complexes in situ, implying that, at rest, $\sim 6 \%$ of VAMP-2 is in a FRET-generating complex with SNAP-25B, presumably as preassembled SNAREs.

Estimating extent of protein overexpression. When cells were transfected with toxin-resistant labeled VAMP-2 and SNAP-25B, native unlabeled proteins were eliminated by pretreatment with botulinum $\mathrm{D}$ and tetanus toxins. The extent of overexpression with exogenous protein was estimated as follows: let $V$ be the total number of uncomplexed VAMP molecules in a region of measurement and $F$ be the fraction of VAMP labeled with cerulean, then FVVAMPs are labeled, $(1-F) V$ are not, and the ratio of labeled to unlabeled VAMPs is $F /(1-F)$, which is the overexpression level. A similar level of overexpression for labeled SNAP-25 was assumed. Let there be $S$ SNAREs in docked vesicles. Without overexpression, there are a similar number of orphan VAMPs (Wienisch and Klingauf, 2006), which we propose to exist as orphan SNARE complexes. Overexpression increases the number of orphan VAMPs (as SNAREs) proportionately (Fernández-Alfonso et al., 2006; Wienisch and Klingauf, 2006), so this adds $S F /(1-F)$ orphan SNARE complexes, for a total of $2 S+S F /(1-F)$ SNAREs. Only dually labeled SNAREs produce FRET, in the amount of $S F^{2}(2+F /(1-F))$. The total number of labeled VAMPs (those complexed in SNAREs and those that are free) is $S F[2+F /(1-$ $F)]+F V$, so $F R_{D}$ should equal $\left[S F^{2}\{2+F /(1-F)\}\right] /[S F\{2+F /(1-F)\}$ $+F V]$ times $\varphi$, the FRET efficiency of dually labeled SNARE complexes. Substituting $F R_{D}=0.0284$ and $\varphi=0.5$ into the above relationship gives $F^{2}-2.0568 F+0.1136+0.0568 \mathrm{~V} / S-0.0568 F V / S=0$.

In cells treated with toxins, only FV VAMPs remain, and they are all labeled. There are $S$ docked SNAREs plus $S$ of the usual orphan SNARE complexes plus somewhat fewer extra orphan SNARE complexes, $S F^{2} /$ $(1-F)$ due to the reduced concentration of VAMP. All SNAREs are dually labeled and undergo FRET. $F R_{D}$ is now $\left[S\left\{2+F^{2} /(1-F)\right\}\right] /[S\{2+$ $\left.\left.F^{2} /(1-F)\right\}+F V\right]$ times $\varphi$, which was measured as 0.0343 , resulting in this equation: $0.9341 F^{2}-1.8627 F+1.8627-0.0686 F V / S+0.0686$ $F^{2} V / S=0$. These equations were solved with MATLAB (MathWorks) to get $F=0.86$ and $V / S=114$, suggesting that $\sim 1 \%$ of VAMPs are in the SNAREs of docked vesicles and that $\sim 7$ times as much is in orphan SNARE complexes when transfection overexpresses protein levels by approximately sixfold.

Statistical analysis. Two-tailed Student's $t$ tests were used for differences between groups or from a given value when distributions passed the Kolmogorov-Smirnov test for normality at $\%$ and the Mann-Whitney test was used when they did not. The Fisher exact test was used for $2 \times 2$ 
a

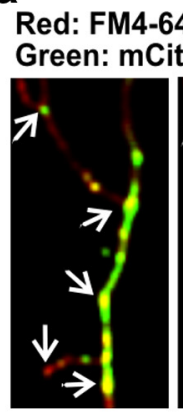

Pre-stim b

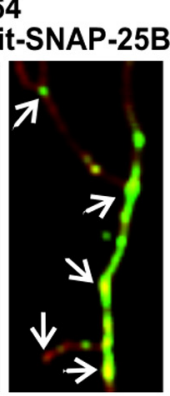

Post-stim

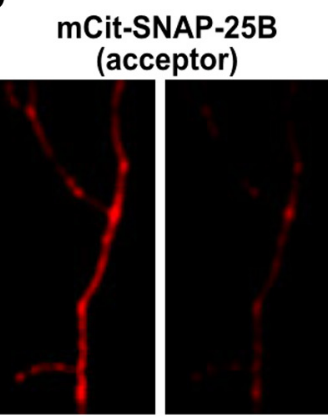

C

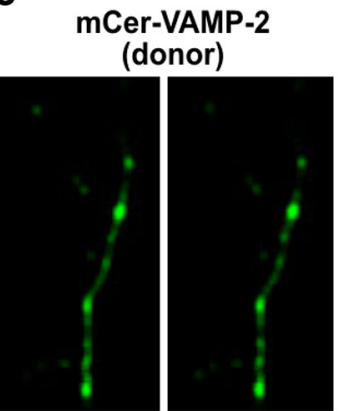

Pre-bleach

Post-bleach

Photobleach acceptor

e

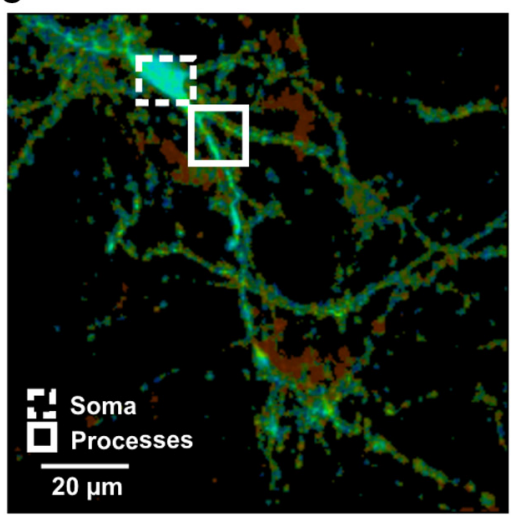

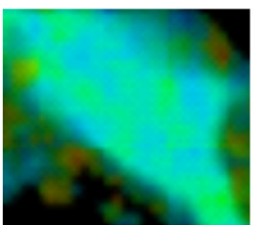

Pre-bleach

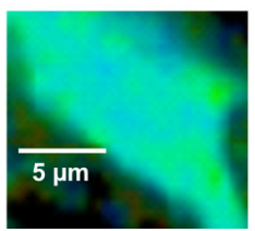

Post-bleach f

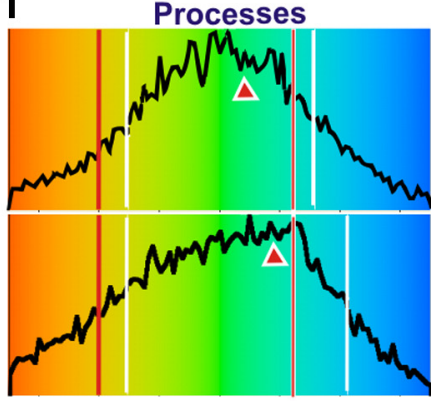

Soma

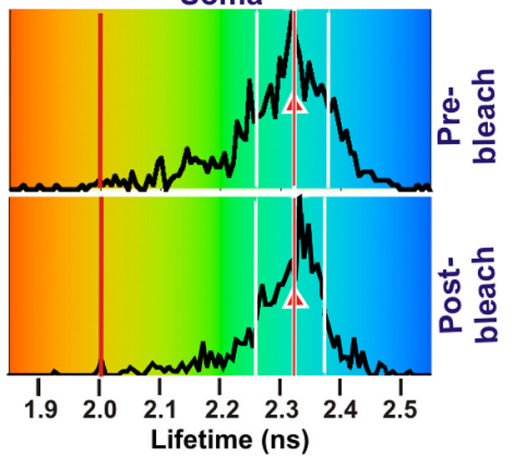

d

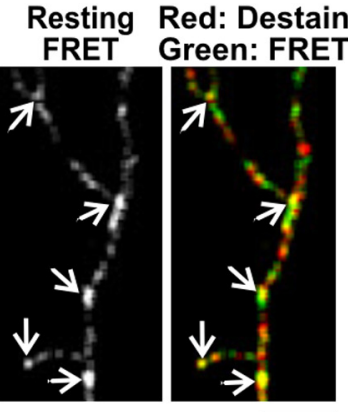

Donor: (post-bleach) - (pre-bleach)
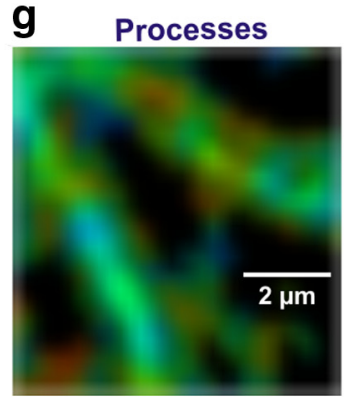

Pre-bleach

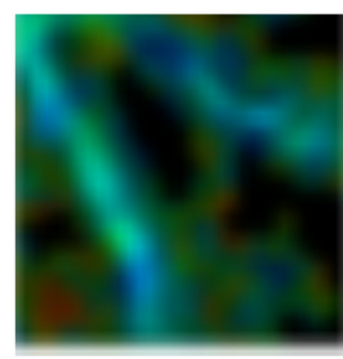

Post-bleach

Figure 2. Resting FRET in neurons transfected with mCer-VAMP-2 and mCit-SNAP-25B. $\boldsymbol{a}-\boldsymbol{d}$, FRET measured by donor dequenching on acceptor photobleaching. $\boldsymbol{a}$, Transmitting synapses in transfected processes (pseudocolored green) identified by FM4 - 64 staining (pseudocolored red) appear as yellow puncta that dim by destaining on stimulation. $\boldsymbol{b}$, mCit-SNAP-25B (pseudocolored red) dims after photobleaching citrine, whereas the mCer-VAMP-2 donor (green) brightens in c. $\boldsymbol{d}$, Increase in donor fluorescence (left) reflects resting FRET with intensity scale expanded 10 -fold from c. Right, this increase is pseudocolored green and overlaid on the decrease in FM4 - 64 fluorescence from a pseudocolored red. Yellow puncta mark transmitting boutons with a resting FRET. $\boldsymbol{e}-\boldsymbol{g}$, FRET measured by FLIM. $\boldsymbol{e}$, Donor fluorescence lifetimes in a dually transfected neuron, color-coded as indicated in $\boldsymbol{f}$. Dashed rectangle marks the soma showing unchanged donor fluorescence lifetimes on acceptor photobleaching (e bottom); solid rectangle marks processes enlarged in $\boldsymbol{g}$ showing a shift to longer (more blue) lifetimes. $f$, Donor lifetime histograms in a region including most processes and in the soma before and after acceptor photobleaching. Red triangles mark peak lifetimes in histograms smoothed by using a 147 ps window and excluding values below dashed red lines, which arise largely from extracellular regions or fine processes contaminated by background fluorescence (orange-red tinge in $\boldsymbol{a}$; Fig. 3); white lines mark histogram half-rise and half-fall times. Resting FRET is indicated by the prolongation of lifetime rise and fall times in processes but not somata after acceptor photobleaching.

comparisons, whereas multiple comparisons such as testing distribution flatness used $\chi^{2}$ tests. Multiple comparisons were done with KruskalWallis tests followed by Dunn's multiple-comparisons test for differences between groups; Student's $t$ tests (after normality testing) or Wilcoxon signed-rank tests (when normality was not satisfied) were used to test for differences from particular values. Unpaired Student's $t$ tests assumed unequal variances; paired tests were used when the same population was tested under different conditions. The test used for each statistical conclusion is given in the text.

The level of uncertainty of FRET calculated from donor fluorescence lifetimes was estimated by the method of propagated uncertainty (Meyer, 1992). When FRET was derived from a ratio of lifetimes, $\tau_{1} / \tau_{2}$, the level of uncertainty, $\sigma$, was estimated from:

$$
\sigma^{2}=\left(\tau_{1} / \tau_{2}\right)^{2}\left[\left(\mathrm{SD} \tau_{1} / \tau_{1}\right)^{2}+\left(\mathrm{SD} \tau_{2} / \tau_{2}\right)^{2}\right]
$$

The uncertainty in FRET derived from a difference between shifts in lifetimes in two groups, $\Delta(\Delta \tau)=(\Delta \tau)_{1}-(\Delta \tau)_{2}$, where group 2 lacked FRET (donor only or soma only), was estimated from:

$$
\begin{aligned}
& \sigma^{2}=\left[\Delta\left(\Delta \tau / \tau_{2}\right]^{2}\left[\left\{\sigma_{\Delta(\Delta \tau)} / \Delta(\Delta \tau)\right\}\right]^{2}+\left(\mathrm{SD} \tau_{2} / \tau_{2}\right)^{2}\right] \\
& \sigma_{\Delta(\Delta \tau)}^{2}=\mathrm{SD}_{(\Delta \tau)_{1}}+\mathrm{SD}_{(\Delta \tau)_{2}}{ }^{2}
\end{aligned}
$$

\section{Results}

Labeled SNAREs function normally

Figure $1 c-e$ shows the expression and correct targeting of VAMP-2 labeled with monomeric cerulean on its $\mathrm{N}$ terminus (mCer-VAMP-2) to clusters of synaptic vesicles in presynaptic boutons and of SNAP-25B labeled with monomeric citrine on its 
$\mathrm{N}$ terminus (mCit-SNAP-25B) to the axonal plasma membrane. To demonstrate that the labeled proteins could still support exocytosis, we used the red amphiphilic dye FM4-64 to stain synaptic vesicles that had undergone exocytosis and endocytosis, and then followed the kinetics of dye destaining in a subsequent stimulus train (Brumback et al., 2004). To ensure that secretion was supported by labeled proteins, we destroyed native SNAP-25B and VAMP-2 with the proteolytic neurotoxins botulinum $\mathrm{E}$ and tetanus toxin (Fig. 1f), and labeled proteins were rendered toxin insensitive by specific amino acid substitutions (Regazzi et al., 1996; Finley et al., 2003). Either of these toxins alone or both together completely blocked neurosecretion in nontransfected cultured rat hippocampal neurons when assayed by FM4-64 staining and destaining (Fig. 1g,h). However, cells cotransfected with toxin-resistant and labeled proteins mCer-VAMP-2(Q76V/F77W) and mCitSNAP-25B(D179K) and treated with both toxins took up FM4-64 dye on stimulation (Fig. 1i) and released it nearly as rapidly as untreated cells when restimulated (Fig. 1j), indicating a nearly normal release probability by action potentials. Identical reductions in release probability were observed in cells transfected with only GFP and in shamtransfected cells (Fig. 1k), indicating that the transfection procedure rather than the attachment of fluorophores reduces release probability by $\sim 35 \%$. This functional rescue demonstrates that exo- and endocytosis are unaffected by labeling SNARE proteins. The procedure also eliminates dilution of FRET by unlabeled native proteins.

\section{Resting FRET of preassembled SNAREs}

Before attempting to detect dynamic FRET signals, we wanted to demonstrate a resting FRET, which should reflect preassembled SNAREs of docked vesicles. We first estimated resting FRET by donor dequench on acceptor photobleach (Miyawaki and Tsien, 2000). This technique eliminates FRET by photobleaching the acceptor (Fig. 2b, mCit-SNAP-25B), resulting in an increase in donor fluorescence (Fig. $2 c$, mCer-VAMP-2) proportional to preexisting FRET. A statistically significant FRET of $4.4 \pm 0.7 \%$ of donor $(p<0.0001$, one-sample $t$ test, $n=14$ boutons from 7 preparations) was largely confined to functional boutons (Fig. 2d), as assessed by FM4-64 staining/destaining (Fig. 2).

More extensive measurements were conducted using FLIM, a method better suited to measuring resting FRET, which reduces the lifetime of the donor fluorophore. Figure $2 e$ is a pseudocolored image of cerulean fluorescence lifetimes in a neuron transfected with mCer-VAMP-2 and mCit-SNAP-25B. Lifetime histograms in the cell body and in a large region, including most processes, are plotted in Figure $2 f$. The lifetimes display wide variability, as is typical of FLIM images. In processes, the peaks of smoothed lifetime histograms were $2.18 \pm 0.036 \mathrm{~ns}$, significantly
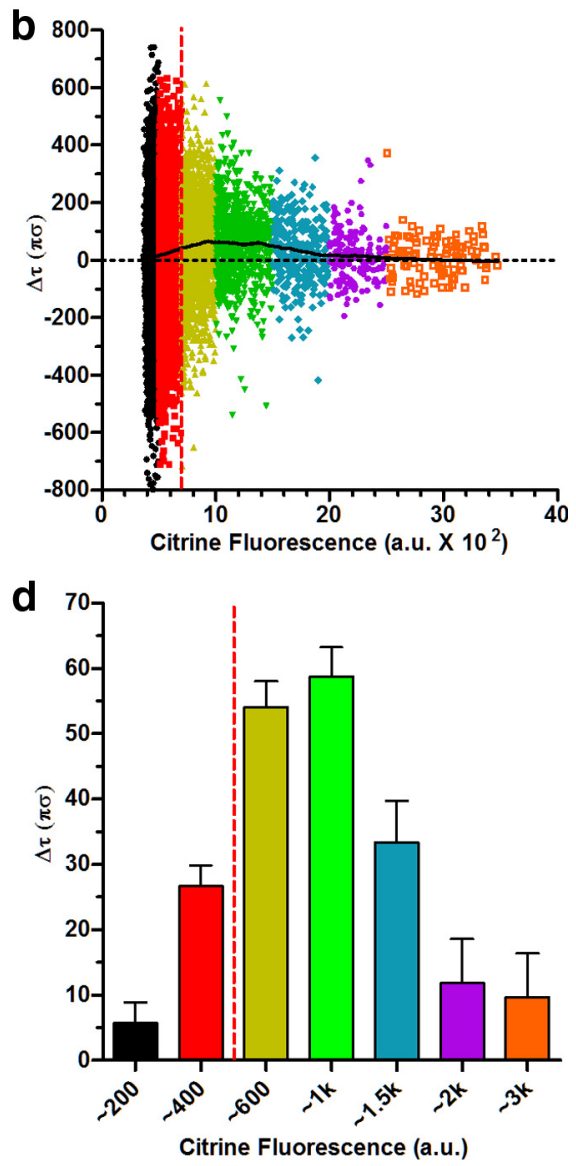

Citrine Fluorescence (a.u.)

Figure 3. Resting FRET is not caused by protein crowding. The distribution of shifts in cerulean fluorescence lifetimes on 列 processes near the soma and nontransmitting (damaged) boutons and also show no FRET. Groups of data were analyzed by

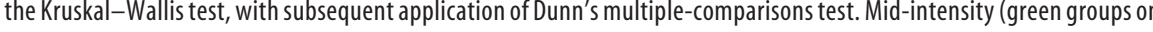
zero $\Delta \tau$, whereas all other groups displayed a significant $\Delta \tau(p<0.0001$ by $t$ test when populations were normal, or by Wilcoxon signed-rank test when they were not).

shorter $(p=0.042, n=5$, paired $t$ test) than lifetimes measured in cell bodies ( $2.24 \pm 0.033 \mathrm{~ns})$, suggesting an average effective FRET ratio of donor of $2.7 \%$ with a propagated uncertainty of $4.9 \%$. We compared distributions of cerulean lifetimes in 6 cells transfected only with the donor to 6 dually transfected neurons. The addition of acceptor reduced average peak donor lifetimes from $2.36 \pm 0.050$ to $2.25 \pm 0.021 \mathrm{~ns}$ ( $p=0.043$, Mann-Whitney test), indicative of an average FRET ratio of VAMP-2 of $4.6 \%$ (5.9\% uncertainty).

We also measured dequenching of FRET in processes from the increase in donor lifetime $(34 \pm 11 \mathrm{ps}, n=13)$ after acceptor photobleaching. In control cells expressing only mCerVAMP-2, mock bleaching of the absent acceptor was followed by a decrease in donor lifetime of $43 \pm 15$ ps $(n=4)$, likely due to donor photoswitching by repeated illumination in this series. The difference was highly significant ( $p=0.0028$, unpaired $t$ test), suggesting an average FRET ratio of VAMP-2 of $3.3 \%$ with an uncertainty of $6.8 \%$. In subsequent experiments, effects of donor photoswitching were eliminated by averaging fewer scans and 
a

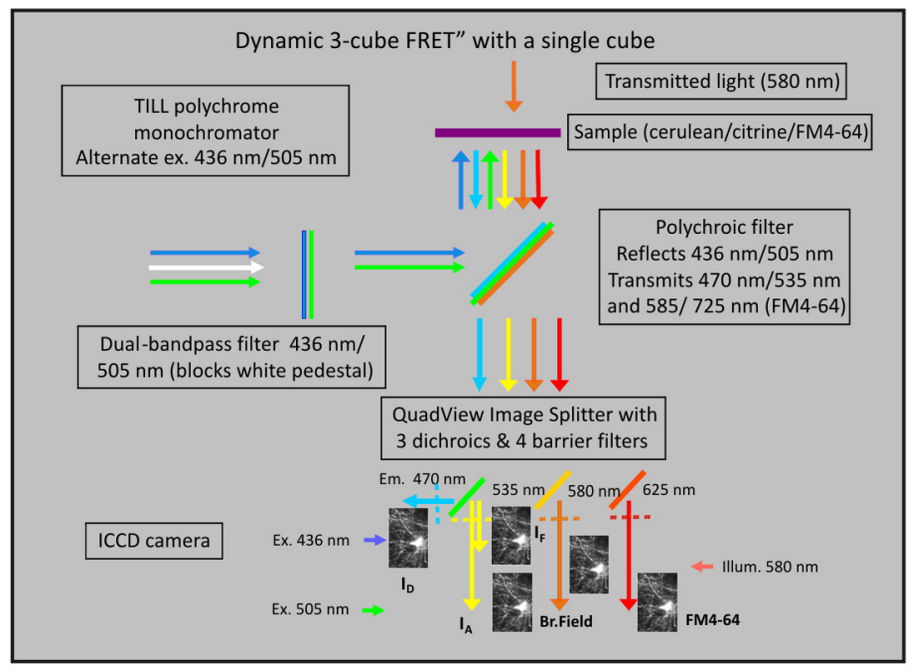

$\mathbf{C}_{1}$

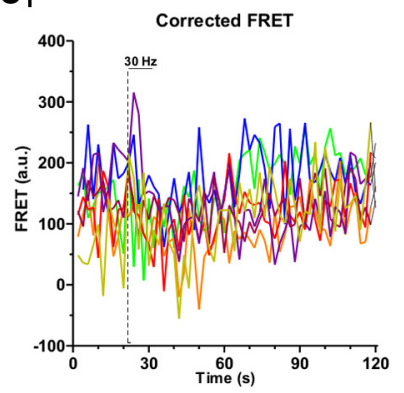

$\mathrm{C}_{2}$

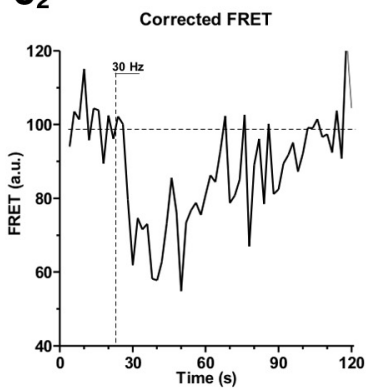

$\mathbf{b}_{1}$
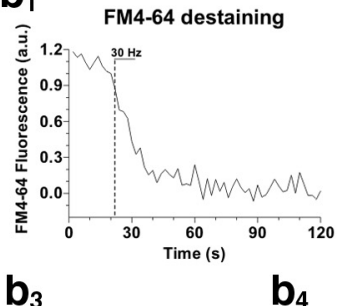

mCit-SNAP25B $\mathrm{O}_{4}$ Corrected FRET

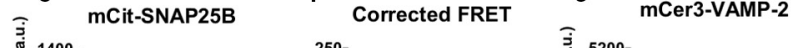

$\mathbf{b}_{2}$

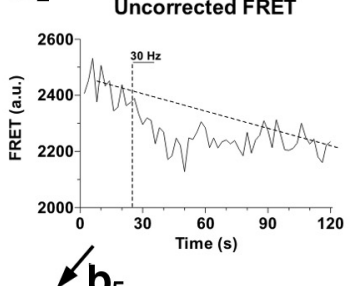

$\mathrm{b}_{5}$

mCer3-VAM

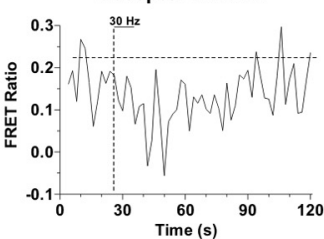

$\mathbf{C}_{3}$

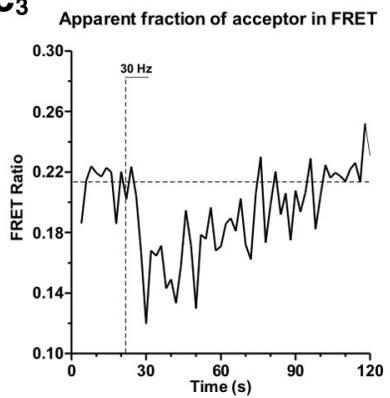

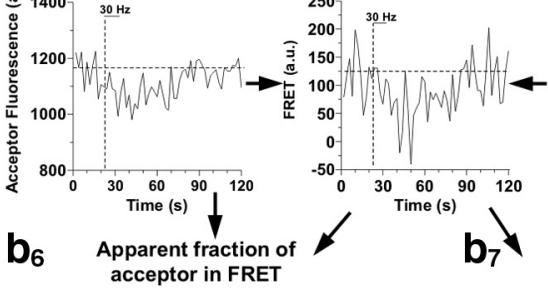

$b_{7}$

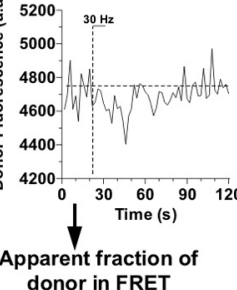

donor in FRET

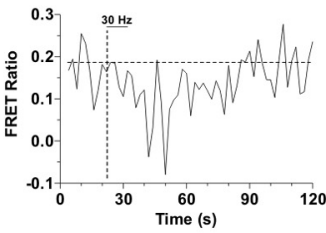

C4 Apparent fraction of donor in FRET

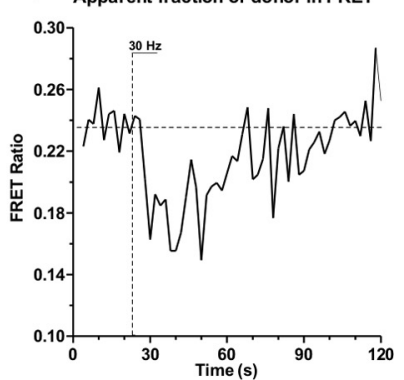

Figure 4. Dynamic FRET between the N termini of SNAP-25B and VAMP-2 measured by enhanced acceptor emission caused by donor excitation at single boutons. $\boldsymbol{a}$, Schema of apparatus used to produce time-lapse image stacks of FRET corrected for spectral spillover. $\boldsymbol{b}$, Time courses of fluorescence measurements from a single bouton used to produce dynamic FRET traces in response to $30 \mathrm{~Hz}$ stimulation. Images used in producing these traces include: $\boldsymbol{b}_{1}$, FM4 - 64 destaining showing functionally transmitting bouton; $\boldsymbol{b}_{2}$, raw FRET from acceptor fluorescence excited at $436 \mathrm{~nm}$, with line used for bleach correction; $\boldsymbol{b}_{3}$, bleach-corrected fluorescence of acceptor excited at $505 \mathrm{~nm}\left(\boldsymbol{b}_{5}\right)$ fluorescence of donor excited at 436 $\mathrm{nm} ; \boldsymbol{b}_{4}$, corrected FRET calculated from $\boldsymbol{b}_{2}, \boldsymbol{b}_{3}$, and $\boldsymbol{b}_{5} ; \boldsymbol{b}_{6}$, FRET ratio of acceptor (apparent fraction of SNAP-25B engaged in FRET), calculated from $\boldsymbol{b}_{3}$ and $\boldsymbol{b}_{4}$; and $\boldsymbol{b}_{7}$, FRET ratio of donor (apparent fraction of VAMP-2 engaged in FRET), calculated from $\boldsymbol{b}_{\mathbf{4}}$ and $\boldsymbol{b}_{5}$. Dashed lines in $\boldsymbol{b}_{2}, \boldsymbol{b}_{\mathbf{3}}$, and $\boldsymbol{b}_{5}$ were used for bleach correction of raw data; dotted lines in $\boldsymbol{b}_{4}, \boldsymbol{b}_{\boldsymbol{6}}$, and $\boldsymbol{b}_{7}$ indicate baseline values. $\boldsymbol{c}$, Dynamic corrected FRET $\left(\boldsymbol{c}_{2}\right)$ and FRET ratios of acceptor $\left(\boldsymbol{c}_{\mathbf{3}}\right)$ and donor $\left(\boldsymbol{c}_{4}\right)$ averaged from 28 boutons showing detectable FRET signals in a single neuron; corrected FRET signals from seven of these boutons are overlaid in $\boldsymbol{c}_{1}$.

pausing between scans for photoswitching artifacts to subside. When we compared (Fig. $2 e, g$ ) average increase in peak donor lifetime on acceptor photobleaching in processes $(60 \pm 22 \mathrm{ps})$ to somata $(6 \pm 13 \mathrm{ps})$, the difference was also significant $(p=$ $0.0017, n=5$, paired $t$ test), suggesting an average FRET ratio of VAMP-2 of $2.4 \%$ (2.5\% uncertainty) restricted to processes. These results indicate a resting FRET ratio of VAMP-2 in processes of $\sim 2.4-4.6 \%$, with most estimates near 3\%. Assuming a cerulean-citrine FRET efficiency of $\sim 0.5$ (see Materials and Methods), this corresponds to $\sim 6 \%$ of presynaptic VAMP-2 complexed as SNAREs. FRET could arise from local crowding of uncomplexed donor and acceptor molecules, in which case lifetimes should decrease with level of expression, as reflected by either donor or acceptor fluorescence intensity. No such dependence was observed (Fig. 3). Changes in lifetime were negligible in the dimmest pixels (arising from extracellular debris and thin processes between boutons) and the brightest pixels (arising mainly from axons near the soma and nontransmitting boutons), but in the intermediate range they were uniform at $\sim 50-60 \mathrm{ps}$ and were independent of the expression level of either donor or acceptor.

Cells treated with botulinum D and tetanus neurotoxins efficiently cleave their target unlabeled proteins, so the fraction of donor-labeled VAMP-2 in SNAREs with acceptorlabeled SNAP-25B should be higher than in cells not treated with toxins. The average peak lifetime increase in toxintreated cells was indeed higher $(38 \pm 14$ ps, $n=8)$ than in untreated cells ( $24 \pm 11 \mathrm{ps}, n=5)$ when acceptor was present, but not significantly. In cells without labeled acceptors, lifetimes decreased $43 \pm 12 \mathrm{ps}$, as mentioned above. As outlined in Materials and Methods, the data suggest that perhaps oneseventh of VAMP-2 or SNAP-25B is unlabeled, that $\sim 1 \%$ of VAMPs are in the SNAREs of docked and releasable vesicles, and that another $7 \%$ are in orphan SNARE complexes. Due to uncertainties in the analysis used to derive these estimates, as well as the high degree of variability in the measurements, we 
have little confidence in the precision of these estimates, but they are interesting because they are consistent with independent estimates of these numbers (see Discussion).

\section{A new dynamic FRET technique}

To detect changes in FRET associated with secretion, we needed to measure dynamically the spatial distribution of small changes in FRET. The most sensitive method uses sensitized emission from an acceptor of energy transferred from an excited donor (Berney and Danuser, 2003; Miyawaki, 2003). Overlap of donor and acceptor fluorescence spectra are required for FRET to occur, so acceptor emission will be contaminated with donor emission and light exciting the donor will also weakly excite the acceptor. These effects are conventionally corrected by measuring $\alpha$, the ratio of fluorescence in the acceptor emission band excited at the donor excitation wavelength divided by acceptor emission excited at the acceptor excitation wavelength in cells transfected with acceptor, and $\beta$, the ratio of acceptor band emission divided by donor band emission, both excited by the donor excitation wavelength in cells transfected with donor. The raw FRET signal, acceptor band emission responding to donor band excitation, measured in successive frames from defined regions of interest, $I_{F}(t)$, is corrected by $I_{F}{ }^{\prime}(t)=I_{F}(t)-\alpha I_{A}(t)-$ $\beta I_{D}(t)$, where $I_{A}(t)$ is the time course of acceptor emission in response to acceptor excitation (which does not excite the donor) and $I_{D}(t)$ is the time course of donor emission in response to donor excitation (pure donor fluorescence; Hoppe et al., 2002). We also calculated a dynamic FRET ratio of acceptor, $F R_{A}(t)=\gamma I_{F}{ }^{\prime}(t) / \alpha I_{A}(t)$, where $\gamma$ is the ratio of acceptor to donor extinction coefficients at the donor excitation wavelength and $F R_{A}(t)$ is proportional to the product of FRET efficiency of the FRET-generating molecules and the proportion of acceptors participating in FRET (Hoppe et al., 2002). We also calculated the analogous FRET ratio of donor, $F R_{D}(t)=I_{F}{ }^{\prime}(t) /\left[I_{F}{ }^{\prime}(t)+\gamma I_{D}(t) / \xi\right]$, where $\xi$ is the ratio of increase in sensitized acceptor emission to decrease in donor fluorescence. See Materials and Methods for our estimation of $\alpha, \beta, \gamma$, and $\xi$. Dynamic FRET ratios provide valuable measures of changes in fraction of FRET partners, but are poor estimators of absolute values of the FRET ratio.

Correcting the FRET signal normally requires three sets of optics: one appropriate for measuring $I_{A}$, one for measuring $I_{D}$, and one for measuring $I_{F}$ by exciting mainly donor and measuring mainly acceptor fluorescence. We developed a dynamic variant of this "3-cube method" in which excitation is switched between two bands using a fast monochrometer, emission of both fluorophores is measured simultaneously using an image splitter with different emission filters, and a polychroic mirror reflects both excitation bands to the preparation while passing

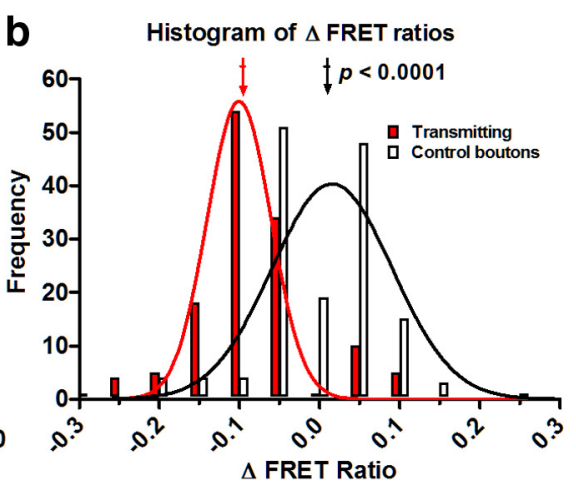

d

Histogram of times to

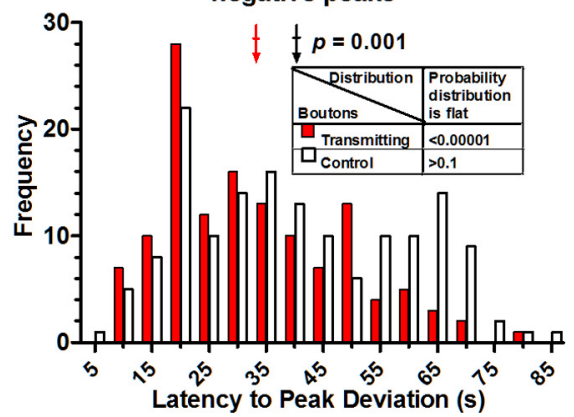

Latency to Peak Deviation (s)

Figure 5. Kinetics of dynamic FRET signals are nonrandom. $\boldsymbol{a}$, Scatter plot of the amplitude of the peak deviations of FRET from 0.007, black arrow and short cross bar). For transmitting boutons, the maximum FRET deviation is almost always negative (mean $\left(p<0.00001, \chi^{2}\right.$ test, $10 \mathrm{df}$, measured between 20 and $65 \mathrm{~s}$ after stimulation began), whereas the nontransmitting bouton histogram is not different from flat $(p>0.1)$.

both emission bands to image splitter and camera. Our polychroic also transmits orange light to produce a bright-field image and red light to monitor FM4-64 emission that is excited by the citrine excitation light. A quadruple image splitter separated donor, acceptor, bright-field, and FM4-64 images (Fig. 4a). Acceptor and FM4-64 images were acquired alternately with donor and FRET images, along with bright-field pictures; the FRET images were processed offline to produce a corrected FRET measurement in the regions of interest.

\section{$\mathrm{N}$-terminal FRET signals from active synapses}

To record meaningful FRET signals, we focused on active boutons transmitting with high release probability. These were identified as well stained FM4-64 puncta in presynaptic processes that subsequently destained rapidly on stimulation after washing off FM4-64. Some experiments were done on cells transfected with toxin-resistant SNARE constructs after treatment with tetanus and botulinum E toxins. Although this should have enhanced the FRET signals by eliminating signal dilution from unlabeled SNAREs, the results were similar, probably because of our relatively high levels of overexpression with labeled 
a
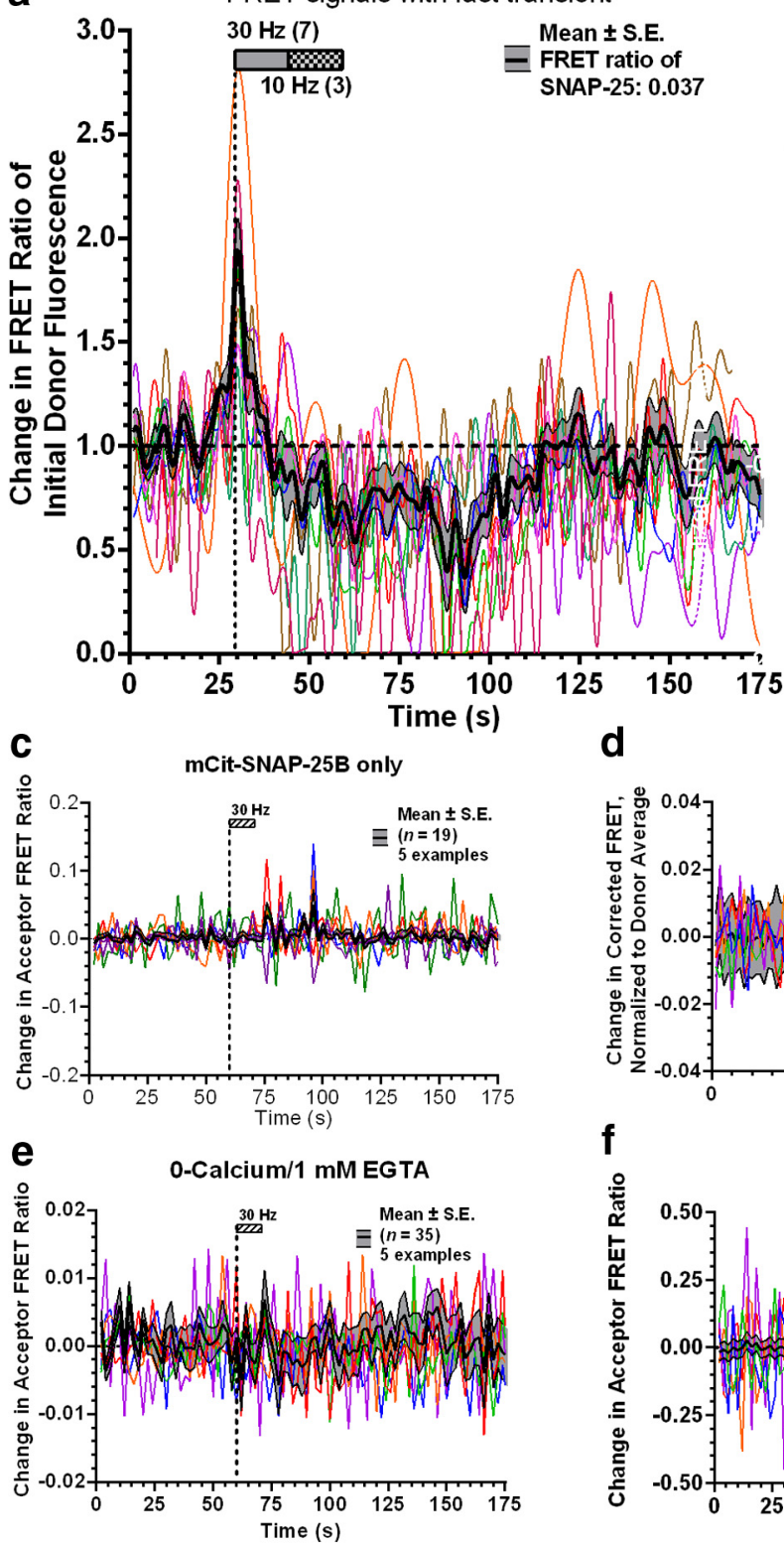

9 After exposure to $\mathrm{N}$-ethylmaleimide

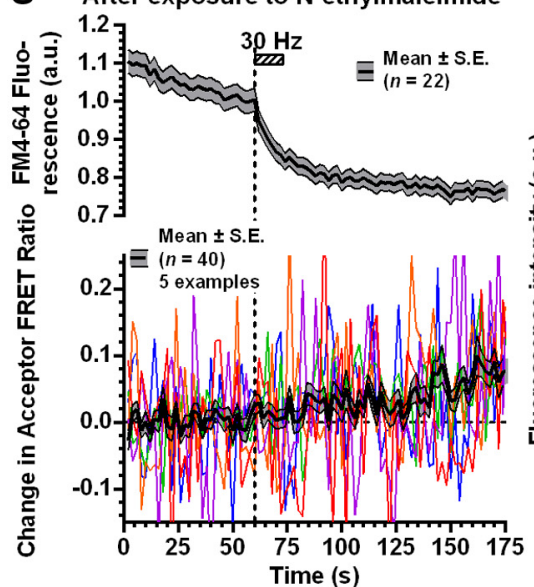

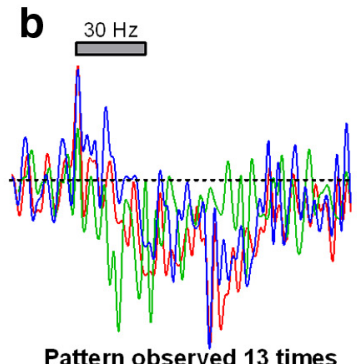

Pattern observed 13 times

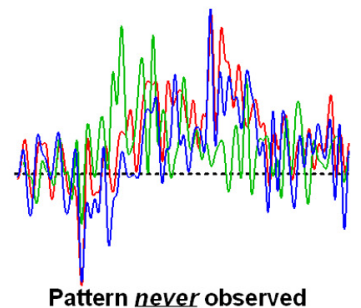

Pattern never observed

\section{d}

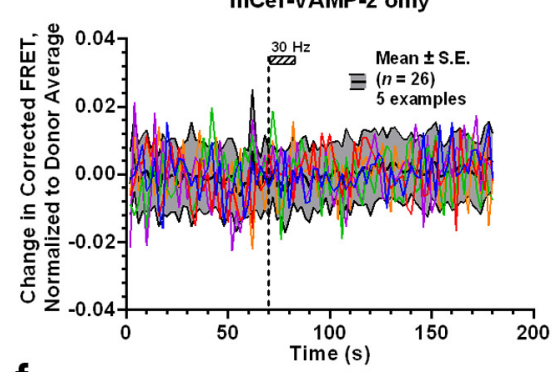

f

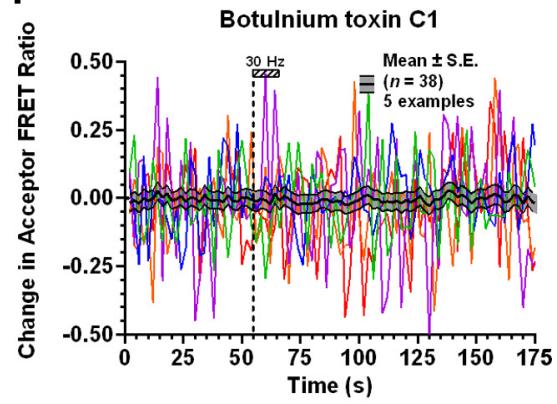

h FM4-64 destaining before NEM, but no restaining after NEM

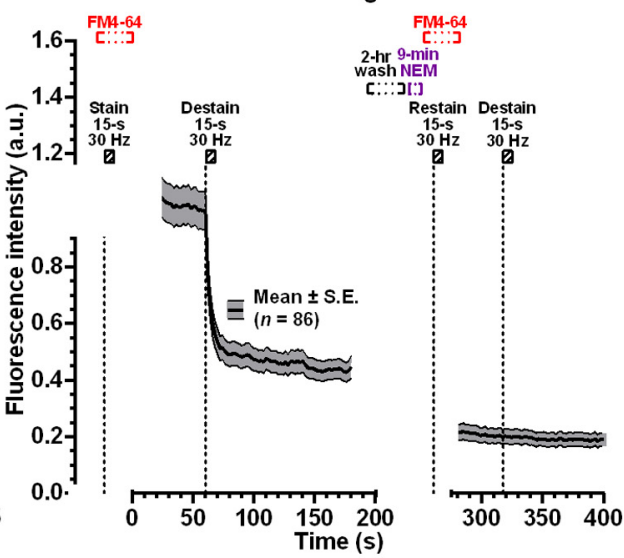

Figure 6. Dynamic N-terminal FRET signals showing a brief increase in FRET at the beginning of stimulation. $\boldsymbol{a}$, Corrected FRET signals (thin colored lines) from 6 boutons stimulated at $30 \mathrm{~Hz}$ and 3 boutons stimulated at $10 \mathrm{~Hz}$ (from 9 different experiments) and the mean (heavy black line) \pm SE (gray zone) of these signals. Corrected FRET is normalized by plotting FRET as a ratio of the

proteins. These results are therefore included with those from experiments without toxin treatment.

Figure $4 b$ presents dynamic fluorescence signals recorded from a single bouton of a neuron subjected to $30 \mathrm{~Hz}$ stimulation for 10 s. Shown are FM4-64 fluorescence, isolated donor (mCer-VAMP-2) fluorescence on exciting cerulean, acceptor (mCitSNAP-25B) fluorescence on exciting only citrine, the raw FRET signal (uncorrected mCit-SNAP-25B fluorescence on exciting cerulean), and the post hoc FRET signals after correction for bleaching, donor emission spillover, and spurious acceptor excitation. The corrected FRET signal is also expressed as dynamic FRET ratios of donor and acceptor.

Figure $4 c$ show numerous examples from one experiment, with averaged corrected FRET and FRET ratio traces. Brief intense stimulation designed to elicit rapid release of the entire readily releasable vesicle pool within $\sim 2 \mathrm{~s}$ (Stevens and Williams, 2007) evoked a gradual decrease in FRET usually beginning $\sim 2-5 \mathrm{~s}$ after the start of stimulation, decreasing to approximately one-half to two-thirds of the initial FRET ratio of acceptor or donor within $20-40 \mathrm{~s}$, and finally recovering to its initial level within another 30-60 s. This pattern of

$\leftarrow$

initial donor fluorescence and then expressing it as a fraction of its average value before stimulation. $\boldsymbol{b}$, Three of the signals from $\boldsymbol{a}$ are repeated in the top panel to illustrate the pattern of FRET signals observed in a total of 13 experiments, whereas the bottom panel shows the inverse of these records showing the pattern that would be expected to occur equally often if the signals in the top panel occurred randomly due to noisy fluctuations. Signals displaying this pattern were never observed. $\boldsymbol{c}-\boldsymbol{f}$, Examples of control experiments showing lack of any discernible FRET ratio of acceptor signals in boutons transfected only with mCit-SNAP-25B (c) or mCer-VAMP-2 (d), or from dually transfected boutons stimulated in a 0 -Ca medium $(\boldsymbol{e})$ or treated with botulinum $\mathrm{C} 1$ toxin $(\boldsymbol{f})$ in cells in which the $m$ Cit-SNAP-25B had been made toxin insensitive by incorporation of the mutation (A199R), leaving only syntaxin-1A sensitive to botulinum C 1 treatment. Changes in the FRET ratios of acceptor from the average prestimulus values are plotted. In $\boldsymbol{g}$, neurons prestained with FM4-64 were exposed to $0.25 \mathrm{~mm}$ NEM for $6 \mathrm{~min}$; subsequent stimulation at $30 \mathrm{~Hz}$ resulted in normal FM4-64 destaining, confirming that exocytosis remained intact but evoked only a slowly increasing FRET ratio of acceptor. In $\boldsymbol{h}$, cells were prestained with FM4 - 64 and then destained, showing intact exocytosis, treated with NEM, and then stimulated again in FM4 - 64; NEM blocked endocytosis and therefore there was no restaining (the decrease in FM4-64 is due to gradual washout of nonspecific staining remaining after destaining before NEM). Because the cells were not stained after NEM exposure, there was no destaining to a fourth period of stimulation. In $\boldsymbol{C}-\boldsymbol{h}$, results averaged from $22-40$ boutons (indicated in each panel) are plotted within \pm one SE band and average FRET signals are overlaid with colored lines representing five typical examples of single-bouton signals. 
a

Prestimulus images

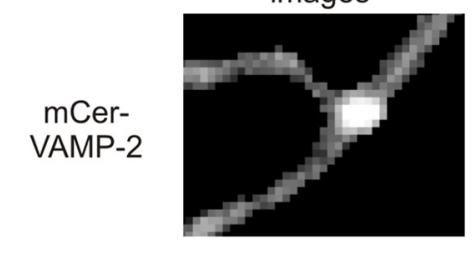

$m$ Cit-

SNAP-25B
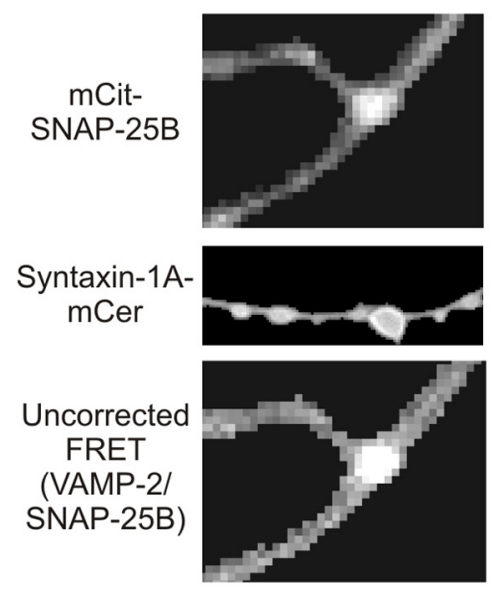
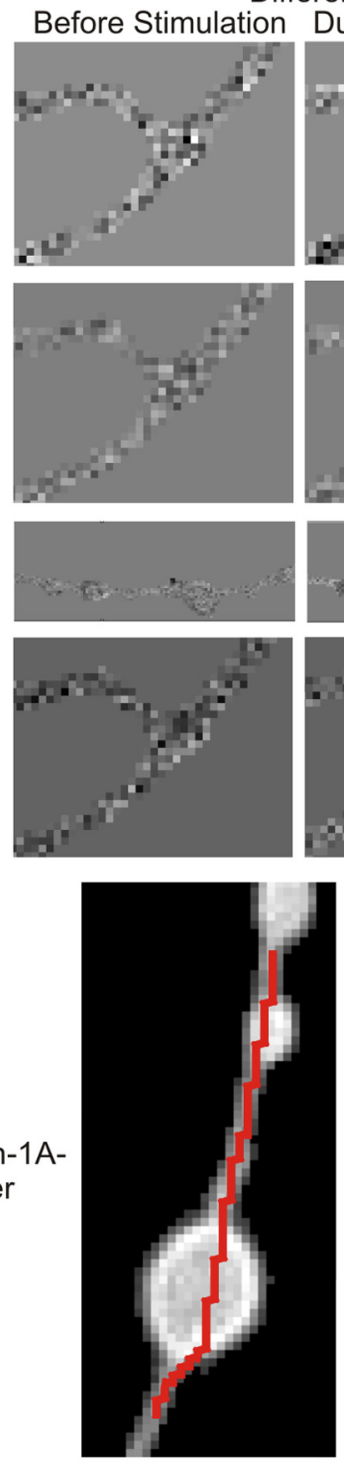

Differential images with respect to pre-stimulation
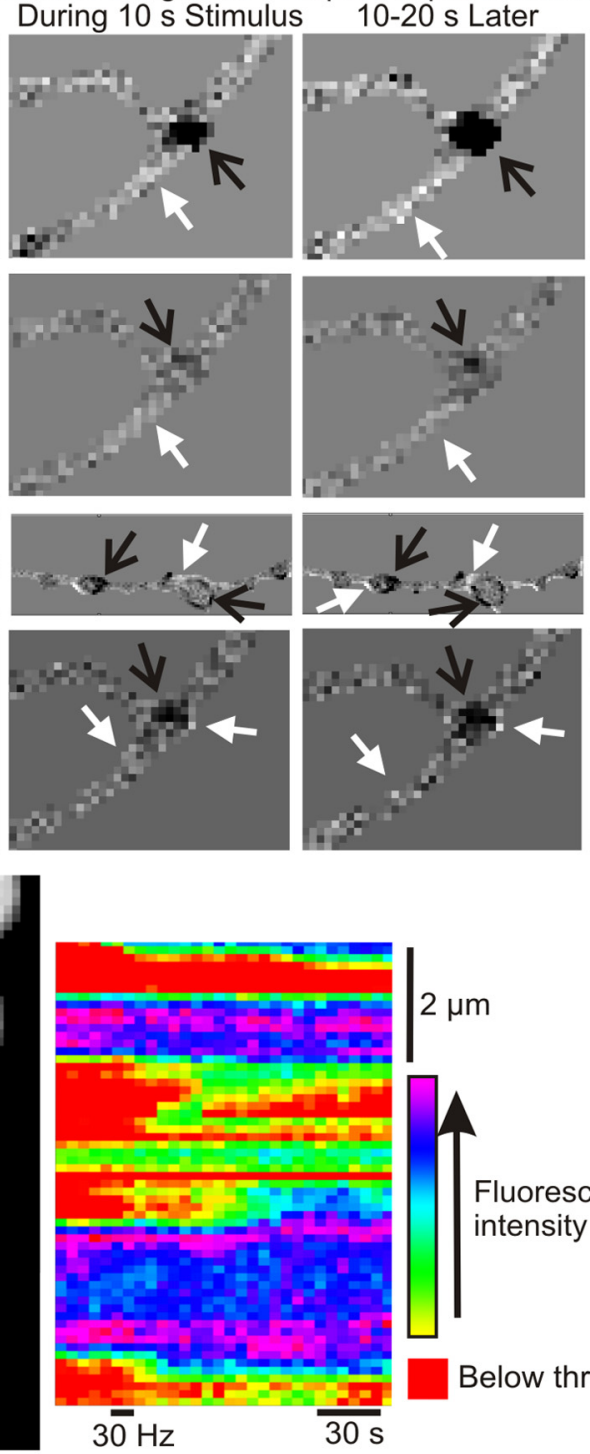

Fluorescence

intensity (a.u.)

Below threshold
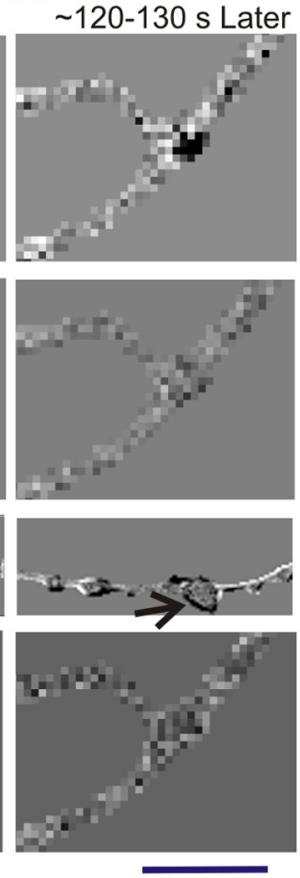

$5 \mu \mathrm{m}$

(7.25 $\mu \mathrm{m}$ for syntaxin)

Figure 7. a, Exemplar images at the times shown before and after stimulation at $30 \mathrm{~Hz}$ for 10 s showing dispersion of mCer-VAMP-2, mCit-SNAP-25B, syntaxin-1A-mCer, and uncorrected FRET between mCit-SNAP-25B and mCer-VAMP-2. All but the syntaxin images were obtained from one bouton at an axonal bifurcation of a dually transfected neuron. For each series, the leftmost image shows fluorescence intensity; the remaining images illustrate changes in intensity from a time before stimulation, with increasing intensity as whiter and decreasing intensity as darker than the gray background. Difference images were $10 \mathrm{~s}$ averages to reduce noise. During and immediately after stimulation, fluorescent protein moves from boutons (which darken, black arrows) to adjacent regions (which brighten, white arrows), with labeled protein distributions gradually recovering. The SNAP-25B movements are also shown in Movie 1.b, Repeated line scans of syntaxin-1A-mCer along the path (red line) through a bouton and neighboring regions shown on the left are displayed on the right, with time plotted horizontally and position plotted vertically. Increasing fluorescence is represented by the color scale plotted on the right. Intensities are in arbitrary units.

FRET signal was observed in 15 experiments on cells from 13 different litters on stimulation at frequencies of 30 (in a few cases, 10) Hz.

The signals in Figure 4 are admittedly noisy; the records from a single bouton are from only $4-8$ pixels. Although the pattern illustrated here was dominant, we also observed examples of records from boutons in which the corrected FRET signal showed a positive peak that was larger than the negative one. The question naturally arises of whether the illustrated pattern is genuinely different from what would be expected to occur from random fluctuations in noisy signals. To address this question, we selected 5 experiments for more detailed analysis (Fig. 5). We recorded 131 single bouton FRET signals from strongly transmitting boutons (assayed by FM4-64 staining/destaining) and plotted the amplitude of the maximum (peak) deviation from initial values, whether positive or negative, versus its time of occurrence for $80 \mathrm{~s}$ from the start of stimulation. We compared these results with FRET records from 152 nontransmitting boutons from the same preparations that were subjected to the same analysis. Peak deviations were identified and measured blindly with an automated computer algorithm to avoid experimenter bias. Peaks from transmitting boutons were predominantly negative (89\%), with an average amplitude of $-8.2 \pm 0.6 \%$ (FRET ratio of initial acceptor), whereas those from nontransmitting boutons were equally likely to be positive $(52 \%)$ or negative $(48 \%)$, with an average FRET ratio of acceptor of $0.3 \pm 0.7 \%$; the differences were highly significant $(p<0.0001$, Fisher exact and unpaired $t$ tests). Moreover, the histogram of latencies of peaks for nontransmitting boutons was flat, with an average time of occurrence exactly at the midpoint of the $80 \mathrm{~s}$ records $(40 \pm 1.5 \mathrm{~s})$, whereas for transmitting boutons, the histogram was peaked, with a 
a

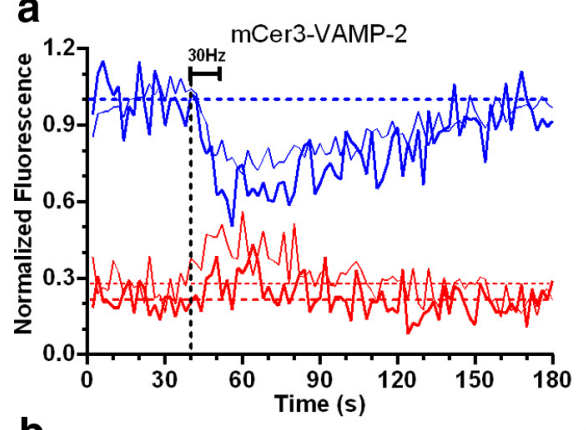

b
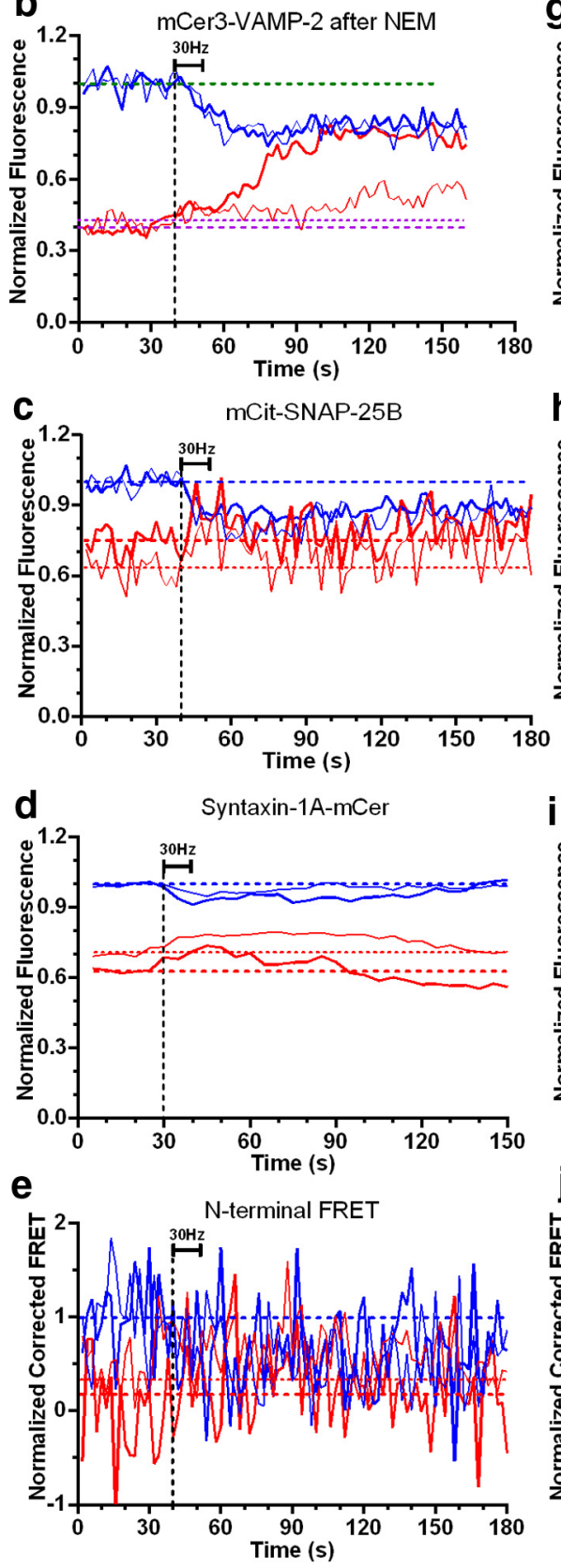
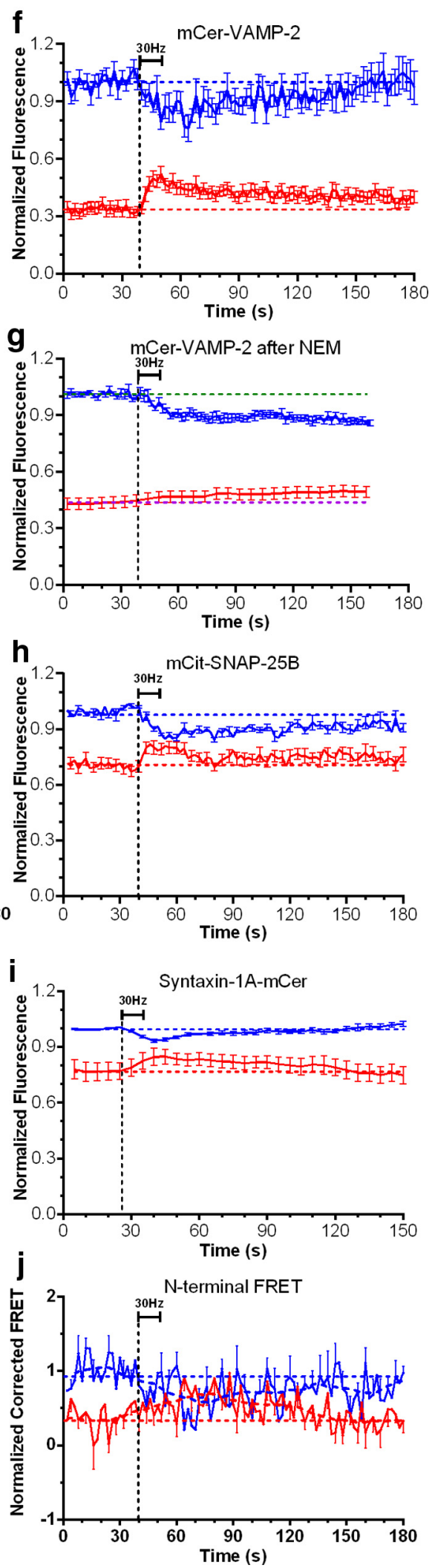

Figure 8. Time courses of fluorescence intensity changes of labeled proteins at active zone centers (blue traces) and surrounds (red traces). $\boldsymbol{a}-\boldsymbol{e}$, Two examples each of movement out of the center and into the surround, respectively, of mCer-VAMP-2, mCer-VAMP-2 after NEM treatment, mCit-SNAP-25B, syntaxin-1A-mCer, and corrected FRET between mCit-SNAP-25B and mCer-VAMP-2. For each bouton, fluorescence is normalized to the average intensity before stimulation in the central region. Averages $\pm S E$ for centers and surrounds from numerous boutons are plotted in $\boldsymbol{f}$ - $\boldsymbol{j}$, with $n=24,43,9,20,8,15,10,12,4$, and 13 , respectively; for surround regions after NEM treatment, 3 adjacent time points were grouped to reduce scatter. Heavy dashed lines injare running averages of 21 points that clearly reveal average changes in noisy records but produce an artifactual early start to temporal changes.

shorter average latency of $33 \pm 1.3 \mathrm{~s}$ from stimulation $(p=0.001$, Mann-Whitney test), and significantly different from flat $\left(p<0.00001, \chi^{2}\right.$ test $)$. Clearly, FRET signals from transmitting boutons are not random and instead fit a consistent pattern, whereas those from nontransmitting boutons do not.

In a few (13) transmitting boutons, we observed a 1-2 s transient increase in FRET right at the beginning of stimulation (Fig. 6a), when almost all of the readily releasable pool of vesicles is released (Stevens and Williams, 2007). Although this transient was only occasionally discernible above the noise, we believe that these signals, too, were real, because in the hundreds of records we have perused, we have never seen the inverse pattern (represented in Fig. $6 b$ by inverting the records we did observe). This difference, too, was significant ( $p=0.0026$, Fisher's exact test).

Several control experiments indicated that these fluorescence signals represented genuine changes in FRET. First, as documented above and illustrated in Figure 5, FRET signals from nontransmitting boutons that showed no FM4-64 destaining did not fit this pattern: the peak change in FRET was equally likely to be positive or negative, to occur at any time in the record, and to have the characteristic shape of a rise time of $\sim 30 \mathrm{~s}$ beginning shortly after stimulation and a fall time of $\sim 60-90 \mathrm{~s}$, as seen consistently in signals from transmitting boutons. Second, no consistent signals at all were observed on stimulating cells transfected with only mCit-SNAP-25B (Fig. $6 c$, acceptor alone), or with only mCer-VAMP-2 (Fig. 6d, donor alone). These results control for possible artifacts due to changes in fluorophore fluorescence and FRET signals arising from other consequences of stimulation, such as changes in $\mathrm{pH}$, oxidation state, or bouton volume. Third, stimulation of cotransfected cells evoked no consistent FRET signals when exocytosis was blocked by omitting calcium from the medium (Fig. 6e), indicating that the FRET signals depend upon an influx of calcium rather than any other effect of stimulation and nerve activity. Fourth, FRET signals could arise from changes in crowding of VAMP-2 and SNAP-25B on the plasma membrane, but the independence of resting FRET on expression level in boutons (Fig. 3) argues against such a source of our FRET signals.

\section{Our FRET signals arise from labeled SNARE proteins}

A more comprehensive control would be to block secretion without blocking cal- 
cium influx to stimulation to show that FRET changes between SNAP-25B and VAMP-2 occur only when SNARE complexes participate in secretion and undergo subsequent disassembly and reassembly. For this purpose we used botulinum $\mathrm{C} 1$ toxin, the primary target of action of which is syntaxin cleavage. This toxin also cleaves native SNAP-25B, so we used SNAP-25B (A199R) mutants that are resistant to botulinum $\mathrm{C} 1$ toxin action (Jin et al., 2007). Cells were cotransfected with mCit-SNAP-25B(A199R) and mCer-VAMP-2 and depolarization loaded with botulinum $\mathrm{C} 1$ toxin. This procedure eliminates SNARE complexes from recycling vesicles by cleaving all uncomplexed syntaxin and syntaxin freed from SNAREs as SNAREs disassemble before vesicle endocytosis while leaving labeled SNAP-25B and VAMP-2 intact. We confirmed that transmission was fully blocked by the absence of FM4-64 staining or destaining on stimulation, which also failed to evoke any change in FRET in these cells (Fig. 6f), confirming that our FRET signals not only require calcium entry but also intact SNAREs. Any effect of stimulation on FRET due to crowding of SNAP-25B and VAMP-2 or to other indirect consequences of stimulation or calcium entry would have persisted in these cells.

To attribute our FRET signals to assembly, disassembly, or other rearrangements of SNARE complexes after electrical stimulation, we sought to alter the normal sequence of events followed by SNAREs in the secretory cycle and look for effects on the FRET signal. For this purpose, we exposed cotransfected neurons to freshly dissolved NEM, a membrane-permeant sulfhydryl alkylating agent that inhibits endocytosis by blocking disassembly of SNAREs by NSF and $\alpha$-SNAP. Because NEM has been reported to have a variety of effects on exocytosis and calcium channels, we first examined concentrations of NEM between 10 and $500 \mu \mathrm{M}$ and found that $0.25 \mathrm{~mm}$ NEM spared exocytosis, as measured by FM4-64 staining before exposure to NEM and destaining after NEM exposure (Fig. $6 g$ ), but blocked endocytosis, as measured by an inability to restain previously stained and destained boutons when applied before a second attempt to stain terminals with FM4-64. NEM treatment eliminated the robust decrease in FRET seen regularly in its absence, suggesting that this phase of the usual FRET signal is associated with the disassembly of SNARE complexes. The remaining slow increase in FRET would then correspond to the assembly of additional SNAREs as new vesicles docked to replace those recently exocytosed. This is perhaps our strongest evidence that our FRET signals arose from the relationship between the $\mathrm{N}$-terminals of SNARE complexes of vesicles undergoing exocytosis and endocytosis.

\section{Dispersion of the SNARE complex}

As described previously (Sankaranarayanan and Ryan, 2000; Li and Murthy, 2001) for labeled VAMP-2, we observed VAMP-2 dispersion from the area of strongest FM4-64 destaining, where vesicles are docked before stimulation, to or past the lateral marregions.

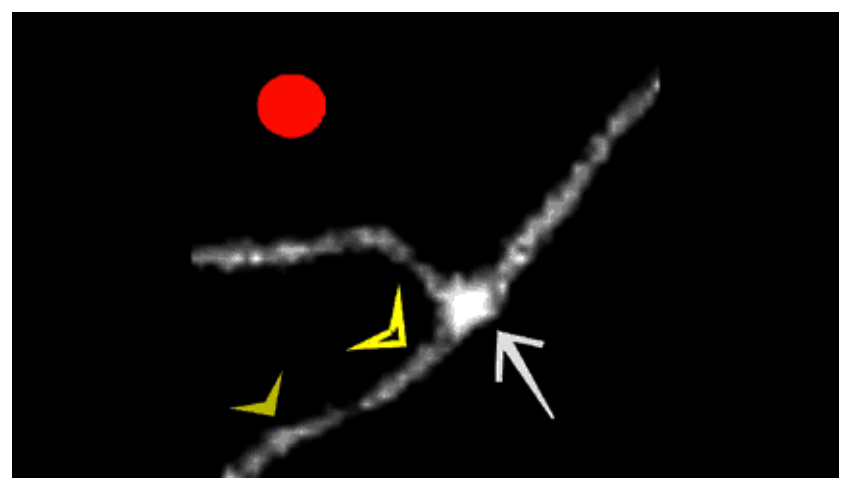

Movie 1. Successive images of mCit-SNAP-25B fluorescence in a presynaptic bouton (at an axonal bifurcation) and surrounding processes. Frames during $10 \mathrm{~s}$ of $30 \mathrm{~Hz}$ stimulation are marked by a red circle. Note the rapid decrease and gradual recovery of SNAP- 25 concentration in the bouton (white arrow) and its dispersion initially into an immediately adjacent region (yellow open arrowhead) and then into more distant regions (yellow closed arrowhead). Each frame is an average of $10 \mathrm{~s}$ of five $0.4 \mathrm{~s}$ frames repeated at $0.5 \mathrm{~Hz}$ (adjusted to optimize signalto-noise). a

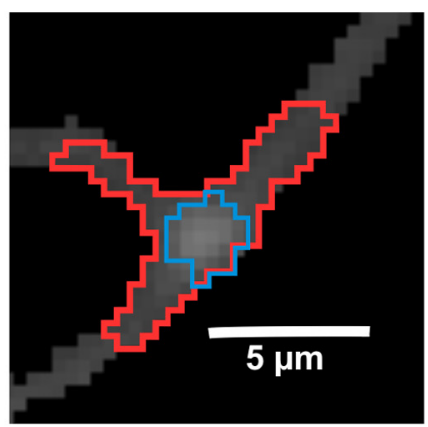

C

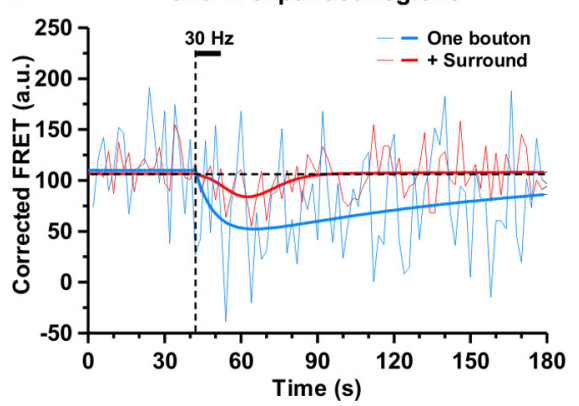

b

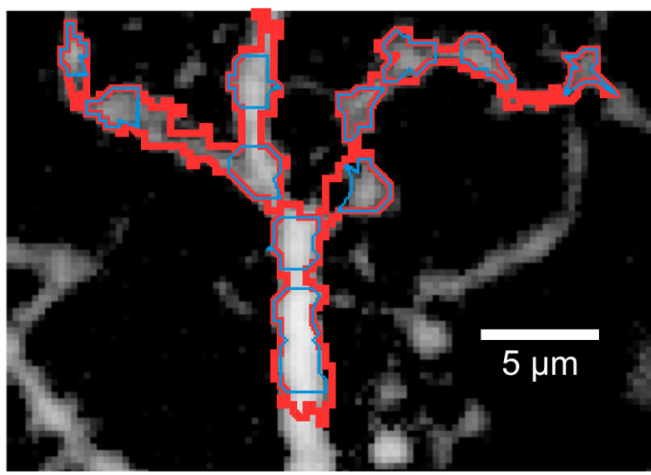

d

Corrected FRET in single boutons and in an axonal section

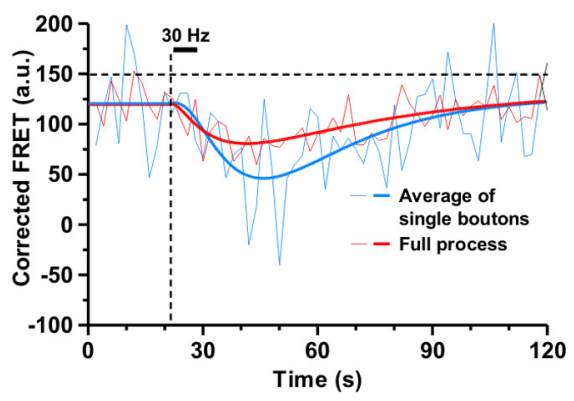

Figure 9. Apparent changes in corrected FRET from N-terminally labeled SNAP-25B and VAMP-2 are due both to dispersion of SNAREs and to real changes in FRET. $\boldsymbol{c}$, When dynamic FRET is measured (thin blue trace) from a single bouton (blue outline in $\boldsymbol{a}$ ), the changes are larger and noisier than when measured from a larger axonal regional surrounding the bouton (thin red trace and red outline in $\boldsymbol{a}$ ). In $\boldsymbol{d}$, signals from 11 boutons (blue outlines in $\boldsymbol{b}$ ) are averaged (thin blue trace) and compared with the signal (thin red trace) from the entire axonal region encompassing all these boutons (red outline in $\boldsymbol{b}$ ). FRET signals from large areas should be devoid of contamination from dispersion of FRET-generating SNAREs out of boutons, which contributes a substantial additional component to the signals from isolated boutons. In $\boldsymbol{c}$ and $\boldsymbol{d}$, the thicker lines are smooth curves drawn through the records (best fitting Boltzmann or double exponential functions), revealing clear differences between boutons with and without surrounding

gins of active zones starting within $1 \mathrm{~s}$ of the beginning of stimulation (Figs. $7 a, 8 a, f)$. This has been interpreted as showing that VAMP-2 is deposited on the plasma membrane on exocytosis and is recovered by endocytosis at the "para-active zone" regions to be recycled to newly docked vesicles in the active zone. After treatment with NEM, VAMP-2 of the secreted vesicles dispersed, 

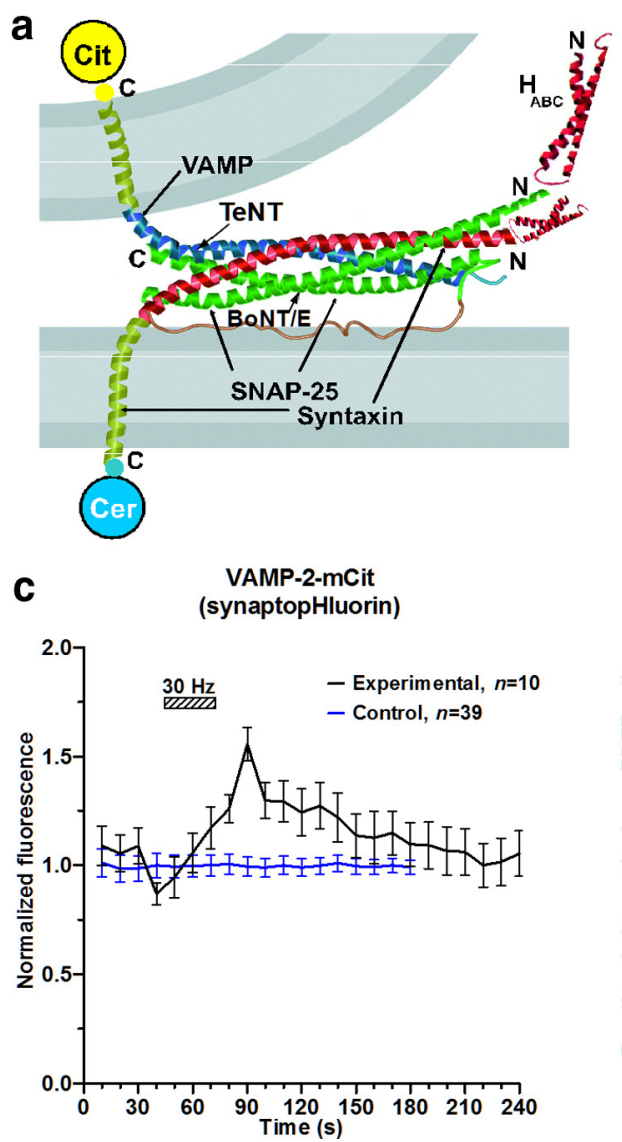

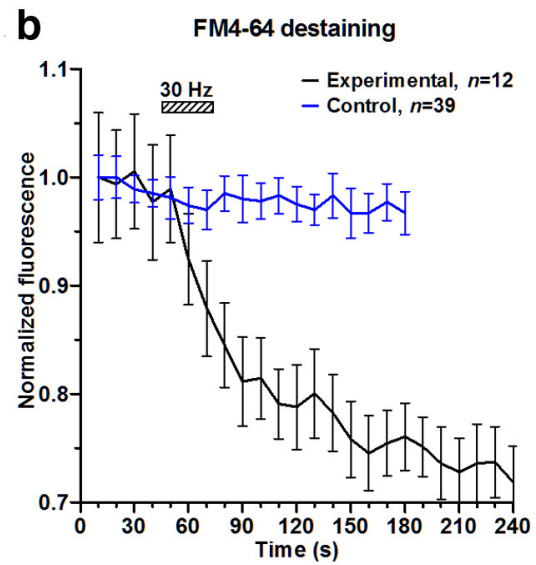

d

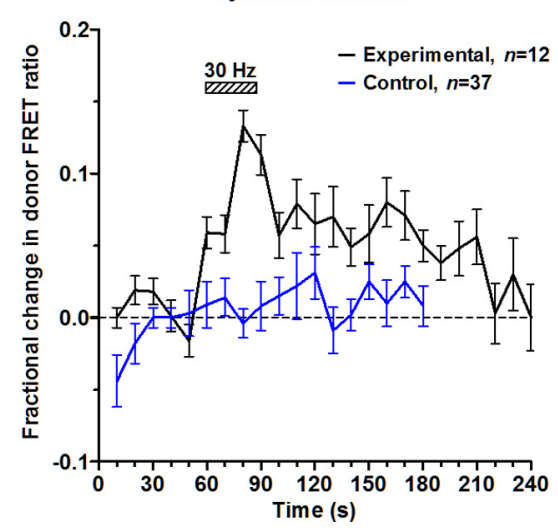

Figure 10. Dynamic FRET between C termini of syntaxin-1A and VAMP-2 measured by donor quenching. $\boldsymbol{a}$, Schematic showing C-terminal labeling of SNARE proteins. $\boldsymbol{b}$, FM4 - 64 destaining allows selection of 12 functionally transmitting synapses (black) and 39 nontransmitting boutons (blue). $c$, The same active synapses produce an increase in VAMP-2-mCit fluorescence on intravesicular alkaline dequenching due to vesicle fusion during $10 \mathrm{~Hz}$ stimulation, which recovers as vesicles are endocytosed and reacidified. $\boldsymbol{d}$, Simultaneous decrease in apparent donor FRET ratio (fraction of syntaxin in FRET multiplied by FRET efficiency), the recovery of which tracks disassembly of cis-SNARES. $\boldsymbol{c}$ and $\boldsymbol{d}$ include null results from nontransmitting boutons. Two each of particularly noisy records were omitted from the experimental VAMP-2-mCit average ( $\boldsymbol{c}$ ) and the control syntaxin-1A-mCer average (d).

but no recovery of VAMP-2 to central active zone regions occurred (Fig. $8 b, g$ ), illustrating that NEM blocked synaptic vesicle recycling but not exocytosis. We have now observed a simultaneous lateral dispersion of both SNAP-25B (Figs. $7 a, 8 c, h$; Movie 1) and syntaxin-1A (Figs. $7 a, b, 8 d, i$ ), and even of the FRET signal itself (Figs. $7 a, 8 e, j$ ), on stimulation. The dynamic dispersion of the target SNARE proteins has not been described previously, although a broader distribution after stimulation has been observed at Drosophila neuromuscular junctions (Kawasaki and Ordway, 2009). It is notable that dispersion appeared to begin before FRET began to decrease (compare Figs. $4 b, c$ and $6 a$, Fig. $8 a-j$ ), suggesting that what we observed was the lateral movement of intact cis-SNARE complexes from newly exocytosed vesicles to sites of subsequent disassembly and endocytosis of vesicle membrane and proteins.

Dispersion of SNARE complexes out of a region of interest will reduce the FRET signal, even if there is no real change in FRET of the moving complex, whereas dispersion of uncomplexed SNAREs could increase the apparent FRET ratio of donor or acceptor. There are three reasons why our changes in FRET signals cannot be attributed entirely to dispersion artifacts: (1) such dispersion cannot explain the regularly observed decrease in the dynamic FRET ratio of both acceptor and donor, $F R_{A}(t)$ and $F R_{D}(t)$, respectively, as the complex moved peripherally (Figs. 4,
6); (2) an artifact arising entirely from dispersion is inconsistent with the reversible decrease in corrected FRET still seen in large regions of interest encompassing active zones and the peripheral regions into which dispersion occurred (Fig. 9); and (3) the absence of a decrease in FRET after NEM treatment while exocytosis and dispersion continued (Figs. $6 g, h, 8 a, f$ ) shows that dispersion alone was not responsible for the changes in the FRET signal. Dispersion contributes to, but by no means entirely accounts for, the corrected FRET signals recorded from central active zone regions. The changes in dynamic FRET ratios, in particular, are strong indications of genuine FRET changes between components of SNARE proteins.

\section{C-terminal FRET}

In a final series of experiments, we looked for the appearance of FRET between VAMP-2-mCit and syntaxin-1A-mCer as a sign of SNARE trans-cis transformation on vesicle fusion (Fig. 10a). Due to the high acidity of synaptic vesicles, the fluorescence of a GFP on the C-terminal of VAMP-2 is strongly quenched. Vesicle exocytosis results in deacidification of the interior vesicle membrane surface, and the sudden dequenching of an intravesicular GFP may be used as a reporter of vesicle fusion-the so-called synaptopHluorin effect (Miesenböck et al., 1998). This phenomenon precludes this label from being used as a FRET reporter, but vesicle fusion should align the $\mathrm{C}$-terminal of VAMP-2 with that of syntaxin-1A on the exterior of the plasma membrane, so a label on its C-terminal might be used to detect FRET. If this extracellular label were citrine, and the intravesicular label were cerulean, then citrine fluorescence would be contaminated by a large, unquantifiable, and uncorrectable spillover from dequenched cerulean emission as it physically approaches the acceptor. To avoid this, we labeled the intravesicular terminal of VAMP-2 with citrine and the extracellular terminal of syntaxin-1A with cerulean, because spillover effects from a $\mathrm{pH}$-dependent change in citrine fluorescence cannot affect the decrease in the shorter wavelength donor fluorescence accompanying FRET. Although this signal is smaller than the increase in acceptor fluorescence by sensitized emission, it does provide a detectable and unambiguous measure of FRET. When strongly transmitting boutons are preselected for strong FM4-64 staining and rapid destaining (Fig. 10b), we detected both an increase in acceptor fluorescence (Fig. 10c, synaptopHluorin effect) and a decrease in donor fluorescence indicating a FRET increase (Fig. $10 d$ ), apparently reporting SNARE trans-cis transformations beginning shortly after the start of $30 \mathrm{~Hz}$ stimulation. The strong synaptopHluorin-like signal reports functional secretion in transfected terminals, although in these experiments, native proteins were not eliminated by neurotoxin treatment combined with toxin-resistant constructs. In contrast, no such fluorescence signals were observed in nontransmitting boutons. This revers- 
ible decrease in donor fluorescence was not produced exclusively by dispersion and replacement of syntaxin, because it was twice as large in dually probetransfected cells $(22.9 \pm 5.3 \%, n=44)$ as in controls transfected only with syntaxin$1 \mathrm{~A}-\mathrm{mCer}(10.0 \pm 2.7 \%, n=39, p=0.034, t$ test; Fig. 11).

Our suggestion of the existence of orphan SNARE complexes based on estimates of resting N-terminal FRET prompted us to look for resting C-terminal FRET. From 7 measurements of syntaxin1A-mCer donor dequenching on VAMP-2mCit acceptor bleaching, we estimate a donor FRET ratio of $10 \pm 6 \%$. Because C terminals of syntaxin and VAMP in the trans configuration are separated by two membranes, this resting FRET is likely another indication of the existence of orphan SNARE complexes, although some assembled intravesicular SNAREs may also contribute to this resting FRET.

\section{The SNARE cycle}

Combining the observations shown in Figures 4 to 10 , we conclude that the following sequence of events most likely underlies our FRET measurements (Fig. 12; Model 1): On stimulation, we sometimes detected a transient increase in N-terminal FRET during exocytosis of docked and primed vesicles, reporting a conformational change or reorientation between the $\mathrm{N}$ termini of VAMP-2 and SNAP-25B, perhaps a tightening or final zippering of the SNARE core complex leading to exocytosis (Chen et al., 2001; Rizo et al., 2006; Sorensen et al., 2006). At the same time FRET appeared between the $\mathrm{C}$ termini of VAMP-2 and syntaxin-1A, reflecting the reorientation of SNAREs from a trans-membrane to a cis-membrane conformation. Fused vesicles began to disperse immediately upon exocytosis, with SNARE complexes still assembled toward active zone margins, whereas before endocytosis, SNAREs were disassembled, causing a reduction in resting $\mathrm{N}$-terminal FRET and a reversal of the increase in C-terminal FRET beginning $\sim 10-20$ $\mathrm{s}$ after the start of stimulation and proceeding for $\sim 45 \mathrm{~s}$ (Ryan and Smith, 1995). Meanwhile, new vesicles arriving at vacated docking sites were primed for release by the (perhaps partial) assembly of SNARE complexes, finally restoring N-terminal FRET to resting levels $\sim 1-2$ min after stimulation while disassembly of released SNAREs was completed (Fig. 10, tail of C-terminal FRET recovery), matching the recovery time course of synaptic transmission from depression (Stevens and Wesseling, 1999). NEM treatment blocked the decrease in N-terminal FRET due to SNARE disassembly, leaving only an apparently permanent increase in FRET as new vesicles docked and assembled their SNAREs, replacing those that fused and dispersed during stimulation.

\section{Discussion}

We report here the first observations of dynamic changes in preassembled SNARE complexes during secretion, their dispersion fluorescence. b Change in cerulean fluorescence normalized to FM4-64 destaining

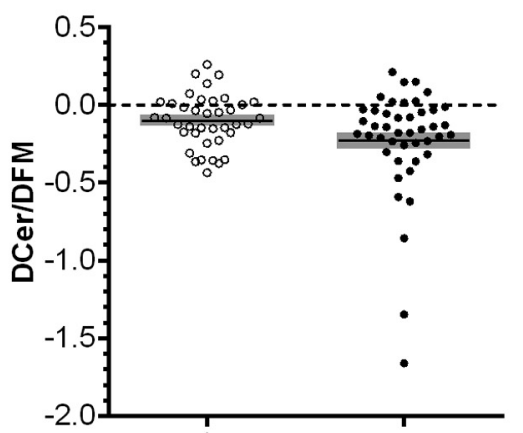

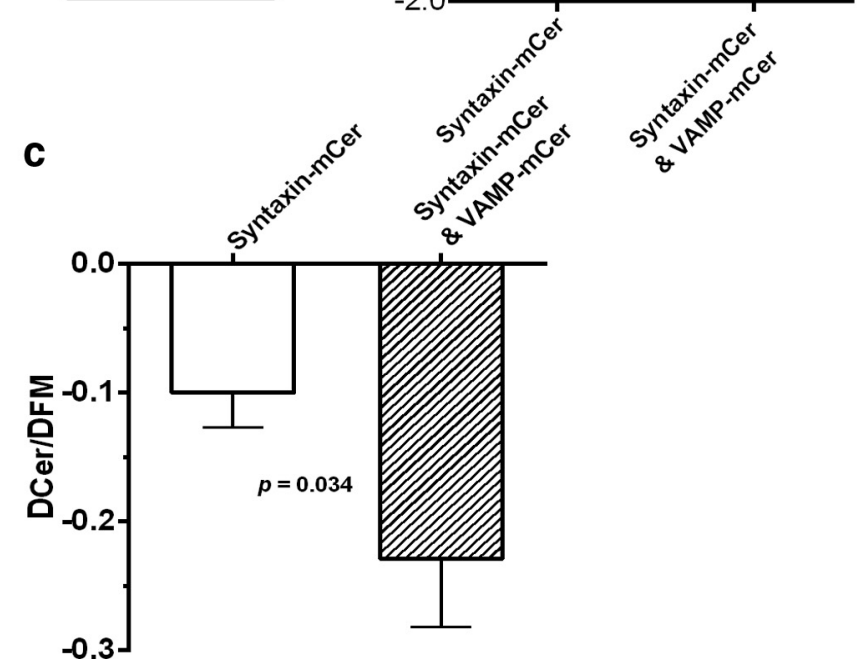

Figure 11. Decrease in syntaxin-1A-mCer fluorescence is due to both dispersion of syntaxin and increase in C-terminal FRET with $\boldsymbol{a}$, Scatter plot of maximum fractional changes in cerulean fluorescence from boutons of dually transfected (closed symbols) and neurons transfected only with syntaxin-1A-mCer (open symbols) in response to $30 \mathrm{~s}$ of $30 \mathrm{~Hz}$ compared with fractional destaining of FM-4 -64 fluorescence in the same boutons. $\boldsymbol{b}$, The results shown in $\boldsymbol{a}$ were changes in FM4 - 64 fluorescence: lines and gray rectangles are means + SE C Means + SE of data in $b$. Both groups show significant decrease in normalized cerulean fluorescence ( $p=0.0007$ for syntaxin only, one-sample $t$ test; $p<0.0001$ for dual transfection, Wilcoxon signed-rank test), and the means are significantly different from each other ( $p=0.034$, $t$ test with Welch's correction), indicating that both syntaxin dispersion and an increase in FRET contribute significantly to the decrease in cerulean

as an intact complex from sites of exocytosis to sites of endocytosis, followed by their disassembly and finally the assembly of SNAREs of newly docked and primed vesicles. Previous measurements of SNARE component interactions using FRET were restricted to static measurements in pheochromocytoma cells (Xia et al., 2001; Liu et al., 2004), except for one study of intramolecular FRET to detect the unfolding of SNAP-25B before the formation of SNARE or SNARE-like complexes (An and Almers, 2004). Although FRET could arise from dimers of only SNAP25B and VAMP-2, such dimers are not likely to form naturally (Brunger, 2006). Our findings are consistent with views that SNAREs assemble (at least partially) before stimulated secretion, undergo conformational rearrangement during fusion, disassemble before endocytosis, and reassemble before subsequent rerelease. In contrast, our findings are more difficult to reconcile with a recently espoused alternative model (Jahn and Fasshauer, 2012) in which exocytosis involves the rapid assembly of fully separated vesicle and plasma membrane SNARE components. Our results also suggest that SNARE complexes disperse intact after fusion and before disassembly. 
a

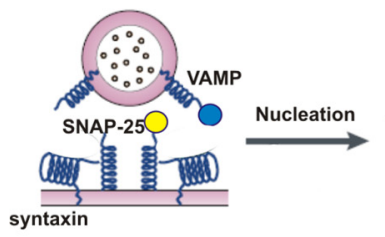

N-terminal labeling
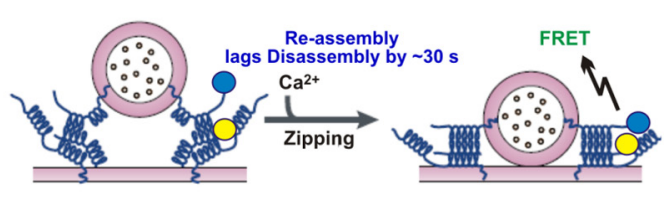

Transient

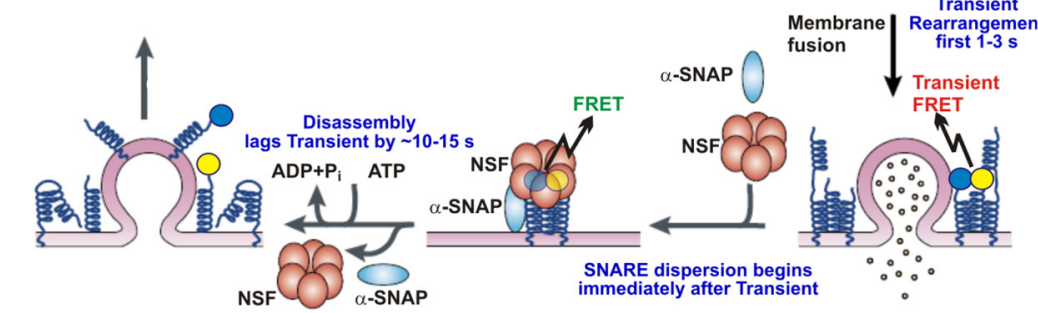

b

\section{C-terminal labeling}

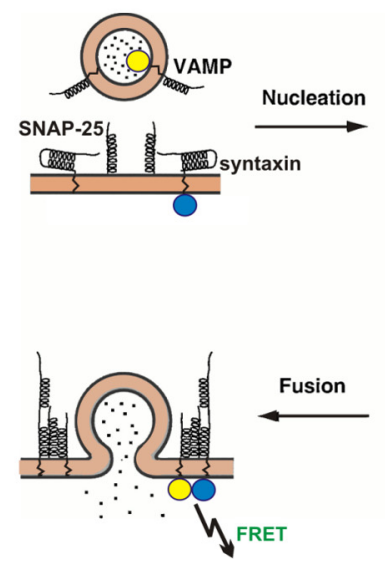

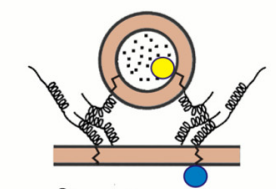

$\mathrm{Ca}^{2+}$

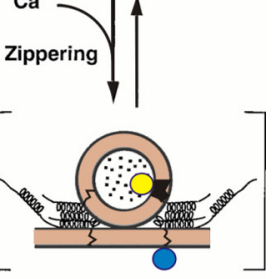

Figure 12. Models of the SNARE cycle and interpretation of our results. $\boldsymbol{a}$, Three phases of dynamic FRET signals (Fig. 6) from N-terminal labeled SNAREs are associated with a model of the SNARE cycle (An and Almers, 2004). During secretion, preassembled SNAREs reorient to generate a briefFRET transient, then disperse before disassembly of recently exocytosed or orphan SNARE complexes (the gradual decrease in FRET), whereas new SNAREs assemble as new vesicles dock and are primed (leading to restoration of FRET). See Model 1 for animated renditions of this sequence. $\boldsymbol{b}$, In C-terminal labeling, an increase in acceptor fluorescence (Fig. 10c) accompanies vesicle fusion and dequenching of intravesicular citrine on VAMP-2 (synaptopHluorin effect), lasting until vesicle endocytosis and reacidification occur. A decrease in donor fluorescence signals an increase in FRET (Fig. 10d) due to the trans-cis transformation of SNAREs on vesicle fusion, lasting until SNAREs are disassembled before endocytosis.

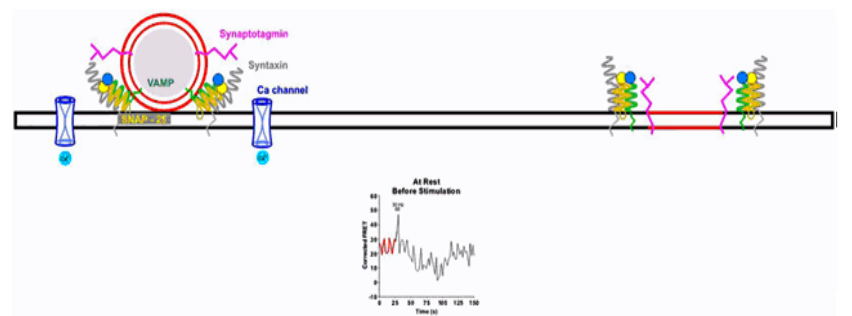

Model 1. Interpretation of the experimental results relating stages in the SNARE cycle in Figure $12 a$ to phases of a corrected FRET signal between N-terminally labeled mCer-VAMP-2 and mCit-SNAP-25B from a single transmitting bouton. Resting FRET arises from assembled SNAREs in docked and primed vesicles and orphan SNARE complexes remaining from prior vesicle exocytosis. Stimulation admits $\mathrm{Ca}^{2+}$ ions through $\mathrm{Ca}^{2+}$ channels that bind to synaptotagmin, causing vesicle fusion sometimes accompanied by a transient increase in FRET. The liberated SNAREs disperse intact to the active zone margins. As they begin to arrive where orphan SNARE complexes are parked, the latter disassemble, leading to a decrease in FRET. FRET eventually returns to resting levels as new vesicles are docked and primed and SNAREs are reassembled either from recovered target SNAREs moving back to the active zone (as shown here) or from free SNAP-25B and syntaxin-1A that were present on the plasma membrane. The increase in C-terminal FRET between VAMP-2-mCit and syntaxin-1A-mCer (Fig. 12b) is not shown, but occurs as SNAREs reorient from trans to cis conformations during vesicle fusion and lasts until orphan SNARE complexes disassemble before endocytosis. Our results cannot distinguish whether it is the SNAREs from newly exocytosed vesicles or orphan SNARE complexes that start to disassemble shortly after stimulation begins. Regardless, our results strongly support the existence of orphan SNARE complexes and suggest that target SNAREs disperse initially intact before disassembly begins.

Our dynamic N-terminal FRET measurements occurred on a background of resting FRET, indicating that $\sim 6 \%$ of the VAMP-2 in a bouton was assembled in SNARE complexes. This proportion was more than we expected because $\sim 10 \%$ of the $\sim 200$ vesicles in an active zone are docked in large, strongly transmitting boutons (Dobrunz and Stevens, 1997; Schikorski and Stevens, 1997), whereas, at most, $10 \%$ of the VAMP-2B in vesicles is likely to be in SNAREs surrounding the incipient fusion pore of docked vesicles (Takamori et al., 2006; Domanska et al., 2009), implying that only $\sim 1 \%$ of VAMP- 2 would be in SNARE complexes. This is close to our estimate of the fraction of VAMP in SNAREs of docked vesicles based on the effect of toxin treatment on the level of resting FRET. Similarly, the majority of
SNAP-25B and syntaxin-1A proteins are located outside of active zones, where they cannot form part of a SNARE complex (TaoCheng et al., 2000). However, a substantial amount of "orphan VAMP"- - several times as much as in the pool of docked vesicles-is left behind on the plasma membrane after a cycle of exocytosis and endocytosis, especially when transfected proteins are somewhat overexpressed; endocytosis recovers mainly this orphan VAMP rather than newly released VAMP (FernándezAlfonso et al., 2006; Wienisch and Klingauf, 2006; Hua et al., 2011). Our high resting $\mathrm{N}$-terminal FRET values, the existence of resting C-terminal FRET, and our analysis of the effect of toxin treatment on resting FRET all suggest that much orphan VAMP-2 remains in the form of "orphan SNARE complexes." It is likely that it is these orphan SNARE complexes whose rapid disassembly was detected (Figs. 4, 6, 10) as new SNAREs arrived from recent exocytosis, providing a "readily retrievable pool" of VAMP-2 for efficient recycling of vesicle membrane and protein into the readily releasable pool and bypassing the reserve pool of vesicles (Hua et al., 2011).

Our results indicate that SNARE disassembly occurs in the endocytic para-active zone region, away from where exocytosis is concentrated. Why is this? We propose that this separate compartmentalization of SNARE disassembly and endocytosis from the site of vesicle docking and release removes the whole array of enzymes and cofactors required for disassembly and endocytosis away from the active zone, which is already overcongested with proteins involved in exocytosis (Fig. 1a,b). The dispersion of SNARE complexes that we observed was probably a manifestation of the process of clearance of "debris" from the active zone that has been proposed to be necessary before active zones can be reused.

The fraction of C-terminal-labeled VAMP resident on the membrane could in principle be estimated by relating its fluorescence to the pool of vesicles released on exocytosis (the increase in acceptor fluorescence) or to the entire pool of VAMP revealed by vesicle alkalinization with ammonia (Wienisch and Klingauf, 2006). However, unlike true synaptopHluorin, citrine is not fully quenched by low vesicular $\mathrm{pH}$, so its resting fluorescence seriously overestimates the size of the orphan VAMP pool. 
The transient increase in FRET sometimes seen during secretion raises interesting questions about its composition; it probably reflects cumulative signals from the $5-10$ vesicles that fuse in the early part of a 10-30 Hz train (Dobrunz and Stevens, 1997; Schikorski and Stevens, 1997). Our sensitivity and time resolution are presently insufficient to determine the duration of signals from single fusion events.

Our results open opportunities for future studies. For example, preventing SNARE disassembly with the amphiphysin SH3 domain (Shupliakov et al., 1997) should also block the decrease in N-terminal FRET, whereas altering the ratio of synaptotagmin isoforms (Wang et al., 2003) might favor "kiss-and-run" fusions also lacking slow phases and perhaps altering the transient increase. Our detection of states of SNARE complexes with FRET provides a new window into understanding SNARE function in synaptic transmission, introduces a new concept of orphan SNARE complexes, and provides new tools (dynamic threeimage FRET corrections and simultaneous measures of secretion by FM dye or synaptopHluorin imaging with one set of optics) for following protein interactions in living cells. Because only small fractions of VAMP-2 and SNAP-25B in a bouton, and even in a single pixel, interacted, we had to use technical innovations in the optical technology and molecular biology of the proteinprobe combinations. Our refinements and optimizations will likely be of general usefulness for revealing protein interactions in real time wherever small fractions of partners interact, especially if those interactions occur rapidly and reversibly in a confined subcellular compartment.

\section{References}

An SJ, Almers W (2004) Tracking SNARE complex formation in live endocrine cells. Science 306:1042-1046. CrossRef Medline

Berney C, Danuser G (2003) FRET or no FRET: a quantitative comparison. Biophys J 84:3992-4010. CrossRef Medline

Brumback AC, Lieber JL, Angleson JK, Betz WJ (2004) Using FM1-43 to study neuropeptide granule dynamics and exocytosis. Methods 33:287294. CrossRef Medline

Brunger AT (2006) Structure and function of SNARE and SNAREinteracting proteins. Q Rev Biophys 38:1-47. CrossRef Medline

Chen YA, Scheller RH (2001) SNARE-mediated membrane fusion. Nat Rev Mol Cell Biol 2:98-106. CrossRef Medline

Chen YA, Scales SJ, Scheller RH (2001) Sequential SNARE assembly underlies priming and triggering of exocytosis. Neuron 30:161-170. CrossRef Medline

Day RN, Booker CF, Periasamy A (2008) Characterization of an improved donor fluorescent protein for Forster resonance energy transfer microscopy. J Biomed Opt 13:031203. CrossRef Medline

Dobrunz LE, Stevens CF (1997) Heterogeneity of release probability, facilitation, and depletion at central synapses. Neuron 18:995-1008. CrossRef Medline

Domanska MK, Kiessling V, Stein A, Fasshauer D, Tamm LK (2009) Single vesicle millisecond fusion kinetics reveals number of SNARE complexes optimal for fast SNARE-mediated membrane fusion. J Biol Chem 284: 32158-32166. CrossRef Medline

Fernández-Alfonso T, Kwan R, Ryan TA (2006) Synaptic vesicles interchange their membrane proteins with a large surface reservoir during recycling. Neuron 51:179-186. CrossRef Medline

Finley MF, Scheller RH, Madison DV (2003) SNAP-25 Ser187 does not mediate phorbol ester enhancement of hippocampal synaptic transmission. Neuropharmacology 45:857-862. CrossRef Medline

Griesbeck O, Baird GS, Campbell RE, Zacharias DA, Tsien RY (2001) Reducing the environmental sensitivity of yellow fluorescent protein. Mechanism and applications. J Biol Chem 276:29188-29194. CrossRef Medline

Han X, Wang CT, Bai J, Chapman ER, Jackson MB (2004) Transmembrane segments of syntaxin line the fusion pore of $\mathrm{Ca}^{2+}$-triggered exocytosis. Science 304:289-292. CrossRef Medline

Hoppe A, Christensen K, Swanson JA (2002) Fluorescence resonance en- ergy transfer-based stoichiometry in living cells. Biophys J 83:3652-3664. CrossRef Medline

Hua Y, Sinha R, Thiel CS, Schmidt R, Hüve J, Martens H, Hell SW, Egner A, Klingauf J (2011) A readily retrievable pool of synaptic vesicles. Nat Neurosci 14:833-839. CrossRef Medline

Jahn R, Fasshauer D (2012) Molecular machines governing exocytosis of synaptic vesicles. Nature 490:201-207. CrossRef Medline

Jiang M, Deng L, Chen G (2004) High $\mathrm{Ca}^{2+}$-phosphate transfection efficiency enables single neuron gene analysis. Gene Ther 11:1303-1311. CrossRef Medline

Jin R, Sikorra S, Stegmann CM, Pich A, Binz T, Brunger AT (2007) Structural and biochemical studies of botulinum neurotoxin serotype $\mathrm{C} 1$ light chain protease: implications for dual substrate specificity. Biochemistry 46:10685-10693. CrossRef Medline

Kawasaki F, Ordway RW (2009) Molecular mechanisms determining conserved properties of short-term synaptic depression revealed in NSF and SNAP-25 conditional mutants. Proc Natl Acad Sci U S A 106:14658-14663. CrossRef Medline

Keller JE, Cai F, Neale EA (2004) Uptake of botulinum neurotoxin into cultured neurons. Biochemistry 43:526-532. CrossRef Medline

Li Z, Murthy VN (2001) Visualizing postendocytic traffic of synaptic vesicles at hippocampal synapses. Neuron 31:593-605. CrossRef Medline

Lin RC, Scheller RH (1997) Structural organization of the synaptic exocytosis core complex. Neuron 19:1087-1094. CrossRef Medline

Liu J, Ernst SA, Gladycheva SE, Lee YY, Lentz SI, Ho CS, Li Q, Stuenkel EL (2004) Fluorescence resonance energy transfer reports properties of syntaxin 1a interaction with Munc18-1 in vivo. J Biol Chem 279: 55924-55936. CrossRef Medline

Markwardt ML, Kremers GJ, Kraft CA, Ray K, Cranfill PJ, Wilson KA, Day RN, Wachter RM, Davidson MW, Rizzo MA (2011) An improved cerulean fluorescent protein with enhanced brightness and reduced reversible photoswitching. PLoS One 6:e17896. CrossRef Medline

Matteoli M, Verderio C, Rossetto O, Iezzi N, Coco S, Schiavo G, Montecucco C (1996) Synaptic vesicle endocytosis mediates the entry of tetanus neurotoxin into hippocampal neurons. Proc Natl Acad Sci U S A 93:1331013315. CrossRef Medline

Meyer SL (1992) Data analysis for scientists and engineers. Evanston, IL: Peer Management Consultants.

Miesenböck G, De Angelis DA, Rothman JE (1998) Visualizing secretion and synaptic transmission with $\mathrm{pH}$-sensitive green fluorescent proteins. Nature 394:192-195. CrossRef Medline

Miyawaki A (2003) Visualization of the spatial and temporal dynamics of intracellular signaling. Dev Cell 4:295-305. CrossRef Medline

Miyawaki A, Tsien RY (2000) Monitoring protein conformations and interactions by fluorescence resonance energy transfer between mutants of green fluorescent protein. Methods Enzymol 327:472-500. CrossRef Medline

Müller CS, Haupt A, Bildl W, Schindler J, Knaus HG, Meissner M, Rammner B, Striessnig J, Flockerzi V, Fakler B, Schulte U (2010) Quantitative proteomics of the Cav2 channel nano-environments in the mammalian brain. Proc Natl Acad Sci U S A 107:14950-14957. CrossRef Medline

Regazzi R, Sadoul K, Meda P, Kelly RB, Halban PA, Wollheim CB (1996) Mutational analysis of VAMP domains implicated in $\mathrm{Ca}^{2+}$-induced insulin exocytosis. EMBO J 15:6951-6959. Medline

Rizo J, Chen X, Araç D (2006) Unraveling the mechanisms of synaptotagmin and SNARE function in neurotransmitter release. Trends Cell Biol 16:339-350. CrossRef Medline

Rizzo MA, Springer GH, Granada B, Piston DW (2004) An improved cyan fluorescent protein variant useful for FRET. Nat Biotechnol 22: 445-449. CrossRef Medline

Rizzo MA, Springer G, Segawa K, Zipfel WR, Piston DW (2006) Optimization of pairings and detection conditions for measurement of FRET between cyan and yellow fluorescent proteins. Microsc Microanal 12: 238-254. CrossRef Medline

Ryan TA, Smith SJ (1995) Vesicle pool mobilization during action potential firing at hippocampal synapses. Neuron 14:983-989. CrossRef Medline

Sankaranarayanan S, Ryan TA (2000) Real-time measurements of vesicleSNARE recycling in synapses of the central nervous system. Nat Cell Biol 2:197-204. CrossRef Medline

Schikorski T, Stevens CF (1997) Quantitative ultrastructural analysis of hippocampal excitatory synapses. J Neurosci 17:5858-5867. Medline

Shupliakov O, Löw P, Grabs D, Gad H, Chen H, David C, Takei K, De Camilli 
P, Brodin L (1997) Synaptic vesicle endocytosis impaired by disruption of dynamin-SH3 domain interactions. Science 276:259-263. CrossRef Medline

Sørensen JB, Wiederhold K, Müller EM, Milosevic I, Nagy G, de Groot BL, Grubmüller H, Fasshauer D (2006) Sequential N- to C-terminal SNARE complex assembly drives priming and fusion of secretory vesicles. EMBO J 25:955-966. CrossRef Medline

Stevens CF, Wesseling JF (1999) Identification of a novel process limiting the rate of synaptic vesicle cycling at hippocampal synapses. Neuron 24: 1017-1028. CrossRef Medline

Stevens CF, Williams JH (2007) Discharge of the readily releasable pool with action potentials at hippocampal synapses. J Neurophysiol 98:3221-3229. CrossRef Medline

Sutton RB, Fasshauer D, Jahn R, Brunger AT (1998) Crystal structure of a SNARE complex involved in synaptic exocytosis at $2.4 \AA$ resolution. Nature 395:347-353. CrossRef Medline

Takamori S, Holt M, Stenius K, Lemke EA, Grønborg M, Riedel D, Urlaub H, Schenck S, Brügger B, Ringler P, Müller SA, Rammner B, Gräter F, Hub JS, De Groot BL, Mieskes G, Moriyama Y, Klingauf J, Grubmller H, Heuser J, et al. (2006) Molecular anatomy of a trafficking organelle. Cell 127:831-846. CrossRef Medline
Tao-Cheng JH, Du J, McBain CJ (2000) Snap-25 is polarized to axons and abundant along the axolemma: an immunogold study of intact neurons. J Neurocytol 29:67-77. CrossRef Medline

Tucker WC, Weber T, Chapman ER (2004) Reconstitution of $\mathrm{Ca}^{2+}$ regulated membrane fusion by synaptotagmin and SNAREs. Science 304: 435-438. CrossRef Medline

Wang CT, Lu JC, Bai J, Chang PY, Martin TF, Chapman ER, Jackson MB (2003) Different domains of synaptotagmin control the choice between kiss-and-run and full fusion. Nature 424:943-947. CrossRef Medline

Weber T, Zemelman BV, McNew JA, Westermann B, Gmachl M, Parlati F, Söllner TH, Rothman JE (1998) SNAREpins: minimal machinery for membrane fusion. Cell 92:759-772. CrossRef Medline

Wienisch M, Klingauf J (2006) Vesicular proteins exocytosed and subsequently retrieved by compensatory endocytosis are nonidentical. Nat Neurosci 9:1019-1027. CrossRef Medline

Xia Z, Zhou Q, Lin J, Liu Y (2001) Stable SNARE complex prior to evoked synaptic vesicle fusion revealed by fluorescence resonance energy transfer. J Biol Chem 276:1766-1771. CrossRef Medline

Zacharias DA, Violin JD, Newton AC, Tsien RY (2002) Partitioning of lipidmodified monomeric GFPs into membrane microdomains of live cells. Science 296:913-916. CrossRef Medline 